\title{
CONVERGENCE OF MARTINGALES WITH A DIRECTED INDEX SET
}

\author{
BY \\ K. KRICKEBERG
}

1. Introduction.. Convergence theorems on martingales of bounded variation $\left({ }^{1}\right)$ have been proved by Doob [4, pp. 319-332 and pp. 354-355] in the case that the index or parameter set is a set of real numbers. Related results have been obtained by Andersen and Jessen [1] in the case of natural numbers as indices. The kind of convergence involved is the convergence almost everywhere, and the indices are considered in their natural order. On the other hand standard examples of the theory of differentiation and an example of Dieudonné [3] show that there is not necessarily convergence almost everywhere in the sense of Moore and Smith if the index set is directed but not totally ordered.

A particular convergence theorem on martingales with a directed index set can be obtained, of course, as a reformulation of the statement that an additive interval function of bounded variation is differentiable almost everywhere. Each random variable of the martingales used here takes only finitely many values. However, even the most general theorem of this kind on generalized interval functions, so-called cell functions, in [6, p. 239] does not cover the case of an additive set function on a so-called de la Vallée Poussin net [11, pp. 486-493], where we have a martingale with natural numbers as indices in which each random variable takes only finitely or countably many values. This case is a particular one of that treated by Andersen and Jessen; it was, in fact, one of the starting points of their theory. Finally a sufficient condition for the convergence almost everywhere of a martingale with a directed index set has been communicated to me by Mr. L. L. Helms, but his theorem does neither imply Doob's or Andersen-Jessen's convergence theorem nor the differentiability of additive interval functions of bounded variation.

The sufficient condition $V_{0}$ given here embraces all cases just mentioned, but also many martingales with not a totally ordered index set, no restriction on the number of values of their random variables, and not satisfying Helms' condition. The condition $V_{0}$ is a generalization of the conclusion of Vitali's covering theorem. It does not refer to the random variables themselves but only to the Moore-Smith sequence of Boolean sigma-algebras underlying the martingale, and it is always satisfied if the index set is totally ordered. How-

Presented to the Society, December 28, 1955 under the title Convergence of nondenumerable martingales; received by the editors April 2, 1956.

(1) For the definition see p. 321 of the present paper. 
ever, only the case that the sequence of these sigma-algebras is increasing has been considered.

No attempt has been made to deal with mean-convergence, too. Convergence theorems of this kind have been completely treated by Helms [7], and within the theory of cell functions already by Pauc [6, pp. 253-255]. In order to avoid restrictions as the existence of a countable cofinal set of indices our convergence notion is the convergence "modulo null sets" or "essential convergence," i.e. the order convergence in the complete lattice of all classes of random variables equal almost everywhere. The simplest way to deal with this kind of convergence is, of course, to consider a strictly positive sigma-finite measure on a Boolean sigma-algebra which is not'necessarily a set algebra, and to replace random variables by so-called "spectral families," "resolutions of identity," or "Ortsfunktionen." In particular the Vitali condition $V_{0}$ is described completely within this "Boolean" theory. The only drawback seems to be that if the martingale is not given as a sequence of random variables but as a sequence of set functions (cf. $\$ \$ 4,5$, and 11 of the present paper), results concerning the singular parts of these set functions as obtained by Andersen and Jessen [1] are no longer available directly. The case of set algebras and of the ordinary pointwise convergence almost everywhere has been considered in $\$ 11$ assuming the existence of a countable cofinal set of indices. The definition of a separable stochastic process and the existence Theorem 11.6 are straightforward modifications of the definition and Theorem by Doob [4, pp. 51-52 and p. 57], on processes with a "continuous" parameter.

With regard to applications outside of probability theory the basic measure is not supposed to be finite.

The goal of the lattice theoretic considerations in $\$ 4$ is above all the Theorem 4.4 that every martingale of bounded variation from above or from below is the difference of two positive martingales, for this theorem, a generalization of the corresponding one on additive interval functions, allows us to prove the convergence theorem in the case of a positive martingale only. However, the fact that the space of all martingales of bounded variation is a conditionally complete vector lattice seems to deserve some interest, too.

The main lemma of this paper, the density Theorem 7.1, is a particular case of the martingale convergence theorem. Its proof is given by a method developed in the previous paper [8, pp. 246-250], which consists in verifying the conditions $F^{+}$and $F^{-}$in $\$ 3$. The martingale convergence Theorem 8.1 itself can be deduced from the density theorem by a technique similar to one used in the theory of differentiation (cf. [6, p. 240]).

The $\$ 10$ on applications to additive cell functions is complementary to the previous paper [8] on nonadditive cell functions. It had already been pointed out in [8, pp. 219 and 277] that the Vitali condition $\boldsymbol{V}$ which requires the possibility of completing disjoint systems of cells to partitions is much too strong 
in the theory of additive cell functions although necessary in the theory of nonadditive ones. In particular $\boldsymbol{V}$ and the still stronger Vitali conditions in other theories of abstract cell functions cannot be applied to prove the differentiability of additive cell functions of bounded variation on a de la Vallée Poussin net. The condition $V_{0}$ given here covers both cases. It is, as $V$, a Vitali condition which does not require the existence of disjoint coverings of a set approximating it from outside, too. The transformation of the results of $\$ 10$ which naturally involve only the "fineness by partitions" to other fineness relations among partitions may be performed as in $[8, \S 3]$.

2. Preliminaries. Let $\mathfrak{B}$ be a Boolean sigma-algebra with unit element $E$ and zero element $O$, and $\mu$ a sigma-finite strictly positive measure on $\mathfrak{B}$. We define a real, finite or infinite $\mathfrak{B}$-measurable function as a so-called resolution of unity or spectral family in $\mathscr{B}$; cf., e.g., [9] $\left({ }^{2}\right)$. As is well known, the space $\mathfrak{F}$ of all such functions is a complete lattice with respect to its natural order $\left({ }^{3}\right)$, and the set of all characteristic functions of elements of $\mathscr{B}$ is a complete sublattice of $\mathfrak{F}$, closed under all lattice operations in $\mathfrak{F}$, and isomorphic to $\mathfrak{B}$. In particular, $\mathfrak{B}$ is complete, and not only sigma-complete. The symbols $\leqq, \vee$, and $\wedge$ will be used to denote order, supremum, and infimum in $\mathfrak{B}, \mathfrak{F}$, and the real line, whereas $\subseteq, \cup$, and $\cap$ will be reserved for set relations and operations. However, we shall of ten write $A B$ instead of $A \wedge B$ if $A, B \in \mathscr{B}$, and $\mathfrak{R M}$ instead of $\mathscr{R} \cap \mathfrak{M}$ if $\mathbb{R}$ and $\mathfrak{M}$ are sets. $A-B$ denotes the difference in $\mathfrak{B}$ in the case $B \leqq A$.

Elements in $\mathfrak{B}$ like $\{f \leqq g\}$ or $\{f<g\}$, where $f, g \in \mathfrak{F}$, are defined as usual. $f=g[A]$ or $f<g[A]$ means that the restrictions of $f$ and $g$ to the element $A$ of $\mathfrak{B}$ satisfy the equation or inequality in question. Integrability of a function $f$ of $\mathfrak{F}$ over an element $A$ of $\mathfrak{B}$ does not include finiteness of $\int_{A} f d \mu$; if.the latter is the case, $f$ is called summable over $A$. Finally, we shall make use of the following conventions: a positive real function is allowed to take the value 0 , and an increasing sequence may still take the same value for two indices one of which is strictly greater than the other.

Let $\mathfrak{A}$ be a Boolean sigma-subalgebra of $\mathfrak{B}$, i.e. closed under countable Boolean operations, these operations coinciding there with those in $\mathfrak{B}$, and with the same unit and zero element. Then if every function belonging to a subset $(B)$ of $\mathfrak{F}$ is $\mathfrak{A}$-measurable, $V(S)$ and $\Lambda \mathscr{S}$ are also. Indeed, $V(S)$ and $\Lambda(B)$ are the supremum and infimum of a countable subset of $\$ S$. Consequently the lattice operations in the space $\mathbb{E}$ of all $\mathfrak{A}$-measurable functions are restrictions to $\mathfrak{F}$ of those in $\mathfrak{F}$, and, in particular, all lattice operations in $\mathfrak{A}$, and not only the countable ones, are restrictions to $\mathfrak{A}$ of those in $\mathfrak{B}$. If $A \in \mathfrak{B}$, the least element of $\mathfrak{A}$ containing $A$ exists and is called the $\mathfrak{A}$-cover of $A$.

A countably additive real function $\phi$ defined on $\mathscr{B}$ is not required to be finite, but we always assume that $\phi(O)=0$.

(2) Notations are explained more in detail in the previous paper [8].

(3) Following N. Bourbaki we use the term "order" instead of "partial order." 
3. Sequences of measurable functions. Unless stated otherwise, every sequence considered here will be a Moore-Smith sequence with a nonempty index-set $\Theta$, directed by a transitive relation $\ll$, and fixed once for all. Therefore, if e.g. $\left(f_{\sigma} ; \sigma \in \Theta\right)$ is such a sequence, we shall of ten only write $\left(f_{\sigma}\right)$ instead. By a Fréchet sequence is meant a sequence with the set of all natural numbers in their natural order as the index-set. A sequence $\left(f_{\sigma} ; \sigma \in \Theta\right)$ of $\mathfrak{B}$-measurable functions is termed a stochastic process.

We shall call a subset $\Delta$ of the index-set $\Theta$ terminal if there is an index $\eta$ such that $\eta \ll \sigma$ implies $\sigma \in \Delta$, and cofinal if its complement in $\Theta$ is not terminal.

We define the limit inferior and limit superior of a stochastic process $\left(f_{\sigma} ; \sigma \in \Theta\right)$ by

$$
\liminf f_{\sigma}=\bigvee_{\tau \in \Theta} \bigwedge_{\tau \in \sigma} f_{\sigma}, \quad \lim \sup f_{\sigma}=\bigwedge_{\boldsymbol{\sigma} \in \boldsymbol{\Theta}} \underset{r \in \sigma}{\bigvee} f_{\sigma \cdot}
$$

If both functions coincide, $\left(f_{\sigma}\right)$ is said to be convergent to $\lim _{\sigma} f_{\sigma}=\lim \inf _{\sigma} f_{\sigma}$ $=\lim \sup _{\sigma} f_{\sigma}$. Corresponding definitions or the consideration of characteristic functions yield limits inferior and superior in $\mathfrak{B}$.

A sequence $\left(\phi_{\sigma} ; \sigma \in \Theta\right)$ of countably additive functions on $\mathscr{B}$ is said to be terminally uniformly bounded if there exists a terminal set $\Delta$ in $\Theta$ such that the set of all numbers $\phi_{\sigma}(A)$ with $A \in \mathfrak{B}$ and $\sigma \in \Delta$ is bounded, and likewise we define terminally uniform boundedness from above or from below. The sequence $\left(\phi_{\sigma}\right)$ is called terminally uniformly absolutely continuous if given any positive number $\epsilon$ there exist a terminal set $\Delta$ in $\Theta$, a positive number $\delta$, and an element $H$ in $\mathfrak{B}$ of finite measure such that $\sigma \in \Delta, A \in \mathfrak{B}$, and $\mu(H A)<\delta$ imply $\left|\phi_{\sigma}(A)\right|<\epsilon$. Substituting $\phi_{\sigma}(A)<\epsilon$ or $-\epsilon<\phi_{\sigma}(A)$ for the last inequality we obtain the definition of terminally uniformly absolute continuity from above or from below. The sequence $\left(\phi_{\sigma}\right)$ is terminally uniformly bounded from above or terminally uniformly absolutely continuous from above if and only if the sequence of the positive variations $\left(\phi_{\sigma}^{+}\right)$is terminally uniformly bounded or terminally uniformly absolutely continuous, respectively, and likewise with the notions "from below." If $B$ has no atoms, every terminally uniformly absolutely continuous sequence is also terminally uniformly bounded.

A stochastic process $\left(f_{\sigma} ; \sigma \in \Theta\right)$ is called terminally uniformly integrable if given any positive $\epsilon$ there exist a terminal set $\Delta$ in $\Theta$, a positive $\gamma$, and an element $H$ in $\mathfrak{B}$ of finite measure such that, with $G_{\sigma}=\left\{\left|f_{\sigma}\right|>\gamma\right\}$ and $G=E-H$, we have $\int_{G_{\sigma}}\left|f_{\sigma}\right| d \mu<\epsilon$ and $\int_{G}\left|f_{\sigma}\right| d \mu<\epsilon$ for every $\sigma$ in $\Delta$. Substituting $f_{\sigma}^{+}=f_{\sigma} \vee 0$ or $f_{\sigma}=\left(-f_{\sigma}\right) \vee 0$ for $\left|f_{\sigma}\right|$ we get the definition of terminally uniform integrability from above or from below.

The following theorem may be proved as in the case of a Fréchet sequence (cf. $[11$, p. 450], and [5, p. 5]).

3.1. A stochastic process $\left(f_{\sigma}\right)$ is terminally uniformly integrable if and only if the sequence of the indefinite integrals $\int_{A} f_{\sigma} d \mu$, each considered as a function of 
$A$ in $\mathfrak{B}$, is terminally uniformly bounded and terminally uniformly absolutely continuous.

The same theorem is true, of course, with the corresponding notions "from above" or "from below."

As a generalization of Fatou's lemma we have [8, p. 228]:

3.2. If the stochastic process $\left(f_{\sigma}\right)$ is terminally uniformly integrable from below, and if $\lim \inf _{\sigma} f_{\sigma}$ is integrable, we have for every $A$ in $\mathfrak{B}$

$$
\int_{A}\left(\lim _{\sigma} \inf f_{\sigma}\right) d \mu \leqq \liminf _{\sigma} \int_{A} f_{\sigma} d \mu,
$$

and under corresponding hypotheses

$$
\underset{\sigma}{\lim \sup } \int_{A} f_{\sigma} d \mu \leqq \int_{A}\left(\limsup _{\sigma} f_{\sigma}\right) d \mu \text {. }
$$

In particular

$$
\begin{aligned}
& \int_{A}\left(\lim \inf f_{\sigma}^{+}\right) d \mu \leqq \lim \inf \int_{A} f_{\sigma}^{+} d \mu,
\end{aligned}
$$

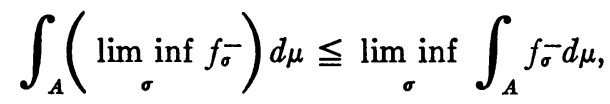

$$
\begin{aligned}
& \int_{A}\left(\lim _{\sigma} \inf \left|f_{\sigma}\right|\right) d \mu \leqq \lim _{\sigma} \inf \int_{A}\left|f_{\sigma}\right| d \mu
\end{aligned}
$$

for every stochastic process $\left(f_{\sigma}\right)$.

As to the problem in which cases equality holds in (3.1)-(3.3) there is the following preliminary result (cf. also 3.6, below):

3.3. If $\left(f_{\sigma}\right)$ is terminally uniformly integrable from below, and $\lim \inf _{\sigma} f_{\sigma}$ integrable, then

$$
-\infty<\int_{E}\left(\lim _{\sigma} \inf f_{\sigma}\right) d \mu=\liminf \int_{E} f_{\sigma} d \mu<+\infty
$$

implies $\int_{A}\left(\lim \inf _{\sigma} f_{\sigma}\right) d \mu=\lim _{\inf } \int_{A} f_{\sigma} d \mu$ for every $A$ in $\mathfrak{B}$.

Under corresponding hypotheses (3.2) or (3.3) become equalities for every A.

Proof. With $B=E-A$ we obtain from 3.2

$$
\begin{aligned}
& \int_{A}\left(\lim \inf f_{\sigma}\right) d \mu \leqq \lim _{\sigma} \inf \int_{A} f_{\sigma} d \mu, \\
& \int_{B}\left(\operatorname{lim\operatorname {inf}f_{\sigma })} d \mu \leqq \liminf _{\sigma} \int_{B} f_{\sigma} d \mu,\right.
\end{aligned}
$$

and therefore 


$$
\begin{aligned}
-\infty & <\int_{E}\left(\lim \inf _{\sigma}\right) d \mu=\int_{A}\left(\liminf _{\sigma} f_{\sigma}\right) d \mu+\int_{B}\left(\liminf _{\sigma} f_{\sigma}\right) d \mu \\
& \leqq \underset{\sigma}{\liminf } \int_{A} f_{\sigma} d \mu+\underset{\sigma}{\lim \inf } \int_{B} f_{\sigma} d \mu \leqq \liminf \left(\int_{A} f_{\sigma} d \mu+\int_{B} f_{\sigma} d \mu\right) \\
& =\underset{\sigma}{\lim \inf } \int_{E} f_{\sigma} d \mu=\int_{E}\left(\liminf f_{\sigma}\right) d \mu<+\infty .
\end{aligned}
$$

Hence equality holds in (3.4).

The terminally uniform integrability of a stochastic process $\left(f_{\sigma}\right)$ does not imply the integrability of $\lim \inf _{\sigma} f_{\sigma}$ or $\lim \sup _{\sigma} f_{\sigma}(\mathrm{cf} .[5, \mathrm{p} .14])$. However, if $\left(f_{\sigma}\right)$ converges, we obtain as in the case of a Fréchet sequence [11, p. 445]:

3.4. Let $\left(f_{\sigma}\right)$ be a convergent sequence of integrable functions, and $f$ its limit. If the sequence of the indefinite integrals $\int_{A} f_{\sigma} d \mu$, each considered as a function of $A$ in $\mathfrak{B}$, is terminally uniformly bounded from below or from above, $f$ becomes integrable, and $-\infty<\int_{E} f d \mu$ or $\int_{E} f d \mu<+\infty$ respectively. $f$ becomes summable if the sequence of these indefinite integrals is terminally uniformly bounded.

In the case of a convergent process we also have

3.5. Let the stochastic process $\left(f_{\sigma}\right)$ converge to the function $f$. Then

$$
\int_{E}|f| d \mu=\lim _{\sigma} \inf \int_{E}\left|f_{\sigma}\right| d \mu<+\infty
$$

implies

$$
\int_{A} f^{+} d \mu=\liminf _{\sigma} \int_{A} f_{\sigma}^{+} d \mu, \quad \int_{A} f^{-} d \mu=\liminf _{\sigma} \int_{A} f_{\sigma}^{-} d \mu
$$

for every $A$ in $\mathfrak{B}$.

Proof. By 3.3 the equation (3.5) implies the same equation with any domain of integration $A$ instead of $E$. By (3.3) we have $\int_{A} f^{+} d \mu \leqq \lim \inf _{\sigma} \int_{A} f_{\sigma}^{+} d \mu$ and $\int_{A} f^{-} d \mu \leqq \lim \inf _{\sigma} \int_{A} f_{\sigma} d \mu$, and therefore

$$
\begin{aligned}
0 & \leqq \int_{A}|f| d \mu=\int_{A} f^{+} d \mu+\int_{A} f^{-} d \mu \leqq \liminf _{\sigma} \int_{A} f_{\sigma}^{+} d \mu+\liminf _{\sigma} \int_{A} f_{\sigma}^{-} d \mu \\
& \leqq \lim _{\sigma} \inf \left(\int_{A^{\prime}} f^{+} d \mu+\int_{A} f_{\sigma}^{-} d \mu\right)=\liminf _{\sigma}\left|f_{\sigma}\right| d \mu=\int_{A}|f| d \mu<+\infty
\end{aligned}
$$

which proves (3.6).

Besides the "terminal" properties of a sequence $\left(\phi_{\sigma}\right)$ or $\left(f_{\sigma}\right)$ as defined above there are the corresponding well known properties, obtained by omitting the adverb "terminally," which refer to the set of all $\phi_{\sigma}$ or $f_{\sigma}$ only, and do not depend on the relation «in $\Theta$. E.g. $\left(\phi_{\sigma}\right)$ is said to be uniformly absolutely continuous if given a positive $\epsilon$ there exists a positive $\delta$ and an element $H$ in $\mathscr{B}$ 
of finite measure such that $\mu(H A)<\delta$ implies $\left|\phi_{\sigma}(A)\right|<\epsilon$ with every $\sigma$ in $\Theta$. This is equivalent to the fact that $\left(\phi_{\sigma}\right)$ is terminally uniformly absolutely continuous relative to every transitive relation $\ll$ in $\Theta$ directing $\Theta$, or, what amounts to the same thing, relative to the "universal" relation $\ll$ in $\Theta$ given by " $\tau \ll \sigma$ for every $\tau$ and $\sigma$ in $\Theta$." Therefore the Theorems 3.1-3.4 remain true if "terminally" is omitted everywhere.'

A stochastic process $\left(f_{\sigma}\right)$ is said to satisfy the condition $F^{+}$if:

$F^{+}:$Every $f_{\sigma}$ and lim sup $f_{\sigma}$ are integrable, and $-\infty<\int_{E} f_{\sigma} d \mu$ holds for cofinally many indices $\sigma$; given any positive number $\epsilon$ and any $\rho$ in $\Theta$ there exists an index $\zeta$ with $\rho \ll \zeta$ such that for any finite subset $\left\{\sigma_{1}, \cdots, \sigma_{p}\right\}$ of $\Theta$ with $\zeta \ll \sigma_{k}(k=1, \cdots, p)$ there is an index $\xi$ with $\zeta \ll \xi$, and $\int_{E}\left(f_{\sigma_{1}} \vee \cdots \vee f_{\sigma_{p}}\right) d \mu$ $\leqq \int_{E} f_{\xi} d \mu+\epsilon$.

Besides $F^{+}$there is, of course, a corresponding condition $F^{-}$which is satisfied by $\left(f_{\sigma}\right)$ if and only if $\left(-f_{\sigma}\right)$ satisfies $F^{+}$.

The following theorem was proved in [8, pp. 231-234]:

3.6. The condition $F^{+}$on the process $\left(f_{\sigma}\right)$ is sufficient for

$$
\int_{A}\left(\lim \sup f_{\sigma}\right) d \mu \leqq \lim \sup \int_{A} f_{\sigma} d \mu
$$

with any $A$ in $\mathfrak{B}$. It is necessary for (3.7) with $A=E$ if $\int_{E}\left(\bigvee_{\tau_{\alpha \sigma}} f_{\sigma}\right) d \mu<+\infty$ for at least one $\tau$ in $\Theta$, and $-\infty<\lim \sup _{\sigma} \int_{E} f_{\sigma} d \mu$.

As a corollary we obtain

3.7. Let $\left(f_{\sigma}\right)$ be a process satisfying $F^{+}$and $F^{-}$, and such that the sequence $\left(\int_{E} f_{\sigma} d \mu\right)$ converges to a finite limit $\alpha$. Then $\left(f_{\sigma}\right)$ converges to a summable limit function $f$, and $\int_{E} f d \mu=\alpha$.

4. Semimartingales and martingales. Let $\left(\mathfrak{B}_{\sigma} ; \sigma \in \Theta\right)$ be an increasing sequence of Boolean sigma-subalgebras of $\mathfrak{B}$, i.e. $\mathfrak{B}_{\tau} \subseteq \mathfrak{B}_{\sigma}$ if $\tau \ll \sigma$. For every $\sigma$, let $\phi_{\sigma}$ be a countably additive real function defined on $\mathfrak{B}_{\sigma}$. The sequence $\Phi=\left(\phi_{\sigma}\right)$ is called a semimartingale relative to $\left(\mathfrak{B}_{\sigma}\right)$ if $\tau \ll \sigma$ and $A \in \mathfrak{B}_{\tau}$ imply $\phi_{\tau}(A) \leqq \phi_{\sigma}(A)$, and a martingale relative to $\left(\mathfrak{B}_{\sigma}\right)$ if under the same assumptions $\phi_{r}(A)=\phi_{\sigma}(A)$ holds. We shall essentially consider only one such sequence $\left(\mathfrak{B}_{\sigma}\right)$, and therefore omit the phrase "relative to $\left(\mathfrak{B}_{\sigma}\right)$ " in most cases. With this convention the sum $\Phi+\Psi=\left(\phi_{\sigma}+\psi_{\sigma}\right)$ of two semimartingales or martingales $\Phi=\left(\phi_{\sigma}\right)$ and $\Psi=\left(\psi_{\sigma}\right)$ is a semimartingale or martingale respectively, provided that $\phi_{\sigma}(A)+\psi_{\sigma}(A)$ has a meaning for every $\sigma$ and every $A$ in $\mathfrak{B}_{\sigma}$. We write $\Phi \leqq \Psi$ if $\phi_{\sigma}(A) \leqq \psi_{\sigma}(A)$ for every $\sigma$ and every $A$ in $\mathfrak{B}_{\sigma}$. A semimartingale $\Phi$ is said to be finite if all numbers $\phi_{\sigma}(A)$ with $\sigma \in \Theta$ and $A \in \mathfrak{B}_{\sigma}$ are finite. In the case of a martingale this is equivalent to the finiteness of $\phi_{\sigma}(E)$; note that $\phi_{\sigma}(E)$ is independent of $\sigma$.

4.1. Let $\Phi=\left(\phi_{\sigma}\right)$ be a semimartingale, and assume that there is an index $\rho$ with $-\infty<\phi_{\rho}(E)$. Define

$$
\tilde{\phi}_{\tau}(A)=\lim _{\tau_{\sharp \sigma}} \phi_{\sigma}(A)=\bigvee_{\tau \ll \sigma} \phi_{\sigma}(A) \quad \text { if } A \in \mathfrak{B}_{\tau} .
$$


Then $\tilde{\Phi}=\left(\widetilde{\phi}_{\tau}\right)$ is the least among all martingales $\Psi$ such that $\Phi \leqq \Psi$.

We call $\tilde{\Phi}$ the integral of $\Phi$, and occasionally also write $\int \Phi$ instead of $\tilde{\Phi}$.

Proof. Clearly $\tau \ll \sigma$ and $A \in \mathfrak{B}_{\tau}$ imply $\tilde{\phi}_{\tau}(A)=\tilde{\phi}_{\sigma}(A)$. Furthermore $\tilde{\phi}_{r}$ is the limit of a monotone increasing sequence of countably additive functions defined on $\mathfrak{B}_{\tau}$ and greater than $-\infty$, namely the restrictions of the $\phi_{\sigma}$ 's to $\mathfrak{B}_{\sigma}$, where $\rho \ll \sigma$ and $\tau \ll \sigma$, and thus $\tilde{\phi}_{\tau}$ itself is countably additive. So $\Phi$ is a martingale, and $\Phi \leqq \tilde{\Phi}$. If $\Psi$ denotes another martingale with $\Phi \leqq \Psi$, then $A \in \mathfrak{B}_{\tau}$ implies $\phi_{\sigma}(A) \leqq \psi_{\sigma}(A)=\psi_{\tau}(A)$ for every $\sigma$ with $\tau \ll \sigma$, hence $\widetilde{\phi}_{\tau}(A)$ $\leqq \psi_{\tau}(A)$.

Let now $\mathfrak{A}$ be a Boolean sigma-subalgebra of $\mathfrak{B}$. We denote suprema and infima in the space $\mathfrak{D}$ of all countably additive real functions defined on $\mathfrak{A}$ by "sup" and "inf." As is well known $D$ is a lattice, and the supremum $\sup (\phi, \psi)$ of two functions $\phi$ and $\psi$ in $D$ is given by

$$
\sup (\phi, \psi)(A)=\mathrm{V}\{\phi(B)+\psi(A-B) ; B \leqq A, B \in \mathfrak{A}\}
$$

if $A \in \mathfrak{A}$, in particular $\phi^{+}=\sup (\phi, 0)$ and $\phi^{-}=\sup (-\phi, 0)$. Moreover the space of all positive functions in $D$ is a complete lattice, and more generally a sufficient condition for the existence of a supremum of a subset $\mathcal{K}$ of $D$ is that $\sup \left\{\phi^{+} ; \phi \in \mathscr{K}\right\}$ or inf $\left\{\phi^{-} ; \phi \in \mathcal{K}\right\}$ remains finite. If $\mathcal{K}$ is directed by $\leqq$ and $-\infty<\phi(E)$ for at least one $\phi$ in $\mathcal{K}$, we have $\left(\sup _{\phi \in \mathcal{K}} \phi\right)(A)$ $=\mathrm{V}_{\phi \in \mathcal{K}}(\phi(A))$ for every $A$ in $\mathfrak{A}$. We now apply these operations for each $\sigma$ to countably additive functions on $\mathfrak{B}_{\sigma}$.

4.2. Let $\mathrm{e}$ be $a$ set of semimartingales. If

$$
\psi_{\sigma}=\sup \left\{\phi_{\sigma} ; \Phi \in \mathfrak{C}\right\}
$$

exists for every $\sigma$, the sequence $\Psi=\left(\psi_{\sigma}\right)$ is the least semimartingale greater than every $\Phi$ in $\mathcal{C}\left({ }^{4}\right)$, and if $\int \Psi$ exists, it is the least martingale greater than every $\Phi$ in $\mathcal{C}$. The semimartingale $\Psi$ exists e.g. in the case that $\mathfrak{C}$ consists only of a finite number of semimartingales. A sufficient condition for the existence of $\Psi$ and $\int \Psi$ is $-\infty<\phi_{\rho}(E)$ for at least one $\Phi$ in $\mathfrak{e}$ and one $\rho$ in $\Theta$. If, moreover, $\mathfrak{e}$ is directed by $\leqq$, we have

$$
\psi_{\sigma}(A)=\bigvee\left\{\phi_{\sigma}(A) ; \Phi \in \mathfrak{C}\right\}
$$

for every $A$ in $\mathfrak{B}_{\sigma}$. Finally if every element of $\mathfrak{C}$ is a martingale, if $\mathfrak{C}$ is directed by $\leqq$, and $-\infty<\phi_{\rho}(E)$ for at least one $\Phi$ in $\mathfrak{C}$ and one $\rho$ in $\Theta$, then the sequence (4.4) is also a martingale, hence in this case $\int \Psi=\Psi$.

Proof. Let $\tau$ and $\sigma$ be fixed with $\tau \ll \sigma$, and denote the restrictions of $\phi_{\sigma}$ and $\psi_{\sigma}$ to $\mathfrak{B}_{\tau}$ by $\phi_{\sigma}^{\prime}$ and $\psi_{\sigma}^{\prime}$. We have $\phi_{\sigma} \leqq \psi_{\sigma}$, thus $\phi_{\sigma}^{\prime} \leqq \psi_{\sigma}^{\prime}$, furthermore $\phi_{\tau} \leqq \phi_{\sigma}^{\prime}$ according to the definition of a semimartingale, hence $\phi_{\tau} \leqq \psi_{\sigma}^{\prime}$ for every $\Phi$ in $\mathrm{C}$. Therefore $\psi_{\tau} \leqq \psi_{\sigma}^{\prime}$, i.e. $\Psi=\left(\psi_{\sigma}\right)$ is a semimartingale. It follows now immediately from the definition of $\psi_{\sigma}$ that $\Psi$ represents the least semimartingale greater than every $\Phi$ in $\mathcal{C}$, and hence, according to $4.1, \int \Psi$ is the least martin-

(4) Cf. [10, p. 30t]. 
gale greater than every $\Phi$ in $\mathcal{C}$ if it exists. The remaining statements of the theorem are obvious.

We denote the set of all semimartingales relative to $\left(\mathfrak{B}_{\sigma}\right)$ by $\$ \mathfrak{T}$, the set of all martingales relative to $\left(\mathfrak{B}_{\sigma}\right)$ by $\mathfrak{N}$, and the sets of all positive semimartingales or all positive martingales by $\delta \mathfrak{T}^{+}$and $\mathfrak{N C}^{+}$, respectively. Suprema and infima in $\$ M$ relative to the order defined above will be indicated by "sup" and "inf," whereas "sup ${ }_{M}$ " and "inf ${ }_{M}$ " will refer to suprema and infima in $\mathfrak{T}$. The lattice operations in $\mathrm{SMC}^{+}$and $\mathfrak{N}^{+}$are, of course, the restrictions of those in $S \mathscr{N}$ or $\mathfrak{M}$ to $S \mathbb{T}^{+}$or $\mathfrak{M C}^{+}$.

4.3. The spaces $\mathrm{STC}^{+}$and $\mathfrak{T}^{+}$are complete lattices, and for every subset $\mathfrak{C}$ of $\mathfrak{T}^{+}$we have $\sup _{M} \mathfrak{C}=\int \sup \mathfrak{C}$ and $\inf _{M} \mathfrak{e}=\inf \mathfrak{C}$. If $\mathfrak{C}$ is a subset of $\mathfrak{T}^{+}$and directed by $\leqq$, we also have $\sup _{M} \mathfrak{C}=\sup \mathfrak{C}$.

Proof. The statements concerning suprema follow immediately from 4.2. The infimum inf $\mathfrak{C}$ of a subset $\mathfrak{C}$ of $s \mathbb{T}^{+}$is the supremum of all positive semimartingales less than every element of $\mathcal{e}$. In the same way the existence of $\inf _{M} \mathfrak{C}$ is established if $\mathfrak{C} \subseteq \mathfrak{M}^{+}$, and clearly $\inf _{M} \mathfrak{C} \leqq \inf \mathfrak{C}$. However $4.1 \mathrm{im}$ plies that $\int \inf \mathfrak{e}$ is also a martingale less than every element of $\mathfrak{e}$, and therefore $\int \inf \mathfrak{e} \leqq \inf _{M} \mathfrak{C}$. On the other hand inf $\mathfrak{e} \leqq \int \inf \mathfrak{e}$, hence $\inf _{M} \mathfrak{e}=\inf \mathfrak{e}$ $=\int$ inf $\mathbb{e}$. This completes the proof.

If $\Phi$ is any semimartingale, $\int \sup (\Phi, 0)$ surely exists by 4.2 . Let now $\Phi$ be a martingale. We call $\Phi^{+}=\int \sup (\Phi, 0)$ the positive variation of $\Phi$, and define likewise the negative variation $\Phi^{-}=\int \sup (-\Phi, 0)$ and the total variation $|\Phi|=\int \sup (\Phi,-\Phi)=\int \sup \left(\Phi^{+}, \Phi^{-}\right)$. Since $\sup (\phi,-\phi)=\sup (\phi, 0)$ $+\sup (-\phi, 0)$ for every countably additive function $\phi, 4.1$ and 4.2 imply

$$
|\Phi|=\Phi^{+}+\Phi^{-} \text {. }
$$

Therefore $\inf _{M}\left(\Phi^{+}, \Phi^{-}\right)=0$ under the assumption that $|\Phi|$ is finite, but this assumption is not superfluous.

The following inequalities are immediate consequences of their analoga for countably additive functions:

$$
\begin{aligned}
& \Phi^{+}-\Psi^{-} \leqq(\Phi+\Psi)^{+} \leqq \Phi^{+}+\Psi^{+}, \\
& \Phi^{-}-\Psi^{+} \leqq(\Phi+\Psi)^{-} \leqq \Phi^{-}+\Psi^{-},
\end{aligned}
$$

provided that these sums and differences have a meaning.

A semimartingale $\Phi$ is said to have bounded variation if there exists a terminal set $\Delta$ in $\Theta$ such that the set of all numbers $\phi_{\sigma}(A)$ with $\sigma \in \Delta$ and $A \in \mathfrak{B}_{\sigma}$ is bounded, and likewise we define bounded variation from above or from below. By (4.1) and (4.3) $\Phi$ is of bounded variation from above if and only if $\int \sup (\Phi, 0)$ is finite, and we can always choose $\Delta=\Theta$ in the definition of "bounded variation from above." Likewise a martingale $\Phi$ has bounded variation or bounded variation from above or from below if and only if respectively $|\Phi|, \Phi^{+}$, or $\Phi^{-}$is finite, and we can always choose $\Delta=\Theta$ in the definition of these three notions. 
4.4. If the martingale $\Phi$ is of bounded variation from above or from below, we have

$$
\Phi=\Phi^{+}-\Phi^{-} .
$$

Proof. Let $\Phi=\left(\phi_{\tau}\right), \Phi^{+}=\left(\tilde{\phi}_{\tau}^{+}\right)$, and $\Phi^{-}=\left(\tilde{\phi}_{\tau}^{-}\right)$. For every index $\tau$, every $\sigma$ with $\tau \ll \sigma$, and every $A$ in $\mathfrak{B}_{\tau}$ we have $\phi_{\tau}(A)=\phi_{\sigma}(A)=\phi_{\sigma}^{+}(A)-\phi_{\sigma}^{-}(A)$, where $\phi_{\sigma^{+}}^{+}=\sup \left(\phi_{\sigma}, 0\right)$ denotes again the positive and $\phi_{\sigma}^{-}=\sup \left(-\phi_{r}, 0\right)$ the negative variation of $\phi_{\sigma}$. The definition of $\Phi^{+}$gives $\tilde{\phi}_{\tau}^{+}(A)=\lim _{\tau \ll \sigma} \phi_{\sigma}^{+}(A)$, similarly $\tilde{\phi}_{\tau}^{-}(A)=\lim _{\tau \ll \sigma} \phi_{\sigma}^{-}(A)$, and therefore $\phi_{\tau}(A)=\tilde{\phi}_{\tau}^{+}(A)-\tilde{\phi}_{\tau}^{-}(A)$ if this difference has a meaning.

Let $\mathfrak{M}_{0}$ be the space of all martingales of bounded variation. The following theorem is a consequence of 4.3, 4.4, (4.5), and (4.6):

4.5. A sufficient but not a necessary condition in order that a subset $\mathfrak{C}$ of $\mathfrak{T}$ has a supremum in $\mathfrak{T}$ is that at least one of the martingales $\sup _{M}\left\{\Phi^{+} ; \Phi \in \mathfrak{C}\right\}$ and $\inf _{M}\left\{\Phi^{-} ; \Phi \in \mathcal{C}\right\}$ is finite. In this case $\sup _{M} \mathcal{C}$ is given by the difference of these two martingales, and the corresponding statement concerning $\inf _{M} \mathfrak{e}$ holds. $\mathfrak{T}_{0}$ is a conditionally complete vector lattice.

Using 4.3 the existence of suprema or infima in $\$ \Re$ could be established under similar assumptions.

All theorems in this section hold, of course, if $\mathfrak{B}$ and the $\mathfrak{B}_{\sigma}$ 's are Boolean algebras only, and if additive functions instead of sigma-additive ones are considered. Such a definition has been used by Bochner [2] for a different purpose. Furthermore $\mathfrak{B}$ need not be a measure algebra since no measure was involved.

5. Integrand representations. Let $\left(\mathfrak{B}_{\sigma}\right)$ be again an increasing sequence of Boolean sigma-subalgebras of $\mathfrak{B}$, and assume that the restriction of $\mu$ to every $\mathfrak{B}_{\sigma}$ is sigma-finite. Then given a semimartingale $\Phi=\left(\phi_{\sigma}\right)$ there exists a stochastic process $\left(f_{\sigma}\right)$ such that $f_{\sigma}$ is $\mathfrak{B}_{\sigma}$-measurable and integrable, and

$$
\phi_{\sigma}(A)=\int_{A} f_{\sigma} d \mu, \quad \text { if } A \in \mathfrak{B}_{\sigma} .
$$

Conversely, if $\left(f_{\sigma}\right)$ is a stochastic process, if $f_{\sigma}$ is $\mathfrak{B}_{\sigma}$-measurable and integrable over $E$, and $A \in \mathfrak{B}_{\tau}$ and $\tau \ll \sigma$ imply $\int_{A} f_{\tau} d \mu \leqq \int_{A} f_{\sigma} d \mu$, the sequence of the $\phi_{\sigma}$ 's defined by (5.1) represents a semimartingale. Therefore we shall also term the process $\left(f_{\sigma}\right)$ a semimartingale, as is usually done. If necessary, $\left(f_{\sigma}\right)$ will be called the integrand representation and $\left(\phi_{\sigma}\right)$ the integral representation of $\Phi$. This applies, of course, in particular to martingales.

The constructions in 4.1 and 4.2 yield the following integral representations $\left(\tilde{\phi}_{\sigma}^{+}\right),\left(\tilde{\phi}_{\sigma}^{-}\right)$, and $\left(\left|\tilde{\phi}_{\sigma}\right|\right)$ of the variations $\Phi^{+}, \Phi^{-}$, and $|\Phi|$ in terms of the integrand representation $\left(f_{\sigma}\right)$ of a martingale $\Phi$ :

$$
\tilde{\phi}_{\tau}^{+}(A)=\lim _{\tau \varangle \sigma} \int_{A} f_{\sigma}^{+} d \mu, \quad \tilde{\phi}_{\tau}^{-}(A)=\lim _{\tau \in \sigma} \int_{A} f_{\sigma}^{-} d \mu,
$$




$$
\left|\tilde{\phi}_{\tau}\right|(A)=\lim _{\tau \otimes \sigma} \int_{A}\left|f_{\sigma}\right| d \mu
$$

if $A \in \mathfrak{B}_{\tau}$. From 4.2, (4.3) it also follows that the supremum in $\$ 9 \pi$ of a set $\mathfrak{C}$ of semimartingales in their integrand representation is given by the sequence $g_{\tau}=\mathrm{V}\left\{f_{\tau} ;\left(f_{\sigma}\right) \in \mathcal{C}\right\}$ if every $g_{\tau}$ is integrable.

Let $\Phi$ be a semimartingale, given both in its integrand representation $\left(f_{\sigma}\right)$ and its integral representation $\left(\phi_{\sigma}\right)$. Then the indefinite integral

$$
\widehat{\phi}_{o}(A)=\int_{A} f_{\sigma} d \mu, \quad A \in \mathfrak{B},
$$

considered as a function of $A$ in $\mathfrak{B}$, gives a natural extension $\widehat{\phi}_{\sigma}$ of $\phi_{\sigma}$ to $\mathfrak{B}$. The notions "terminally uniformly bounded" and "terminally uniformly absolutely continuous," applied to the sequence $\left(\widehat{\phi}_{\sigma}\right)$, have a meaning, and by' 3.1 the terminally uniform integrability of $\left(f_{\sigma}\right)$ can also be expressed in terms of $\left(\widehat{\phi}_{\sigma}\right)$. It is, however, sometimes convenient to describe these notions in terms of the given sequence $\left(\phi_{\sigma}\right)$ only. This is to some extent achieved by the following definitions and theorems.

If $\Delta$ is a terminal set in $\Theta$, the boundedness of all $\phi_{\sigma}(A)$ with $\sigma \in \Delta$ and $A \in \mathfrak{B}_{\sigma}$ is equivalent to the boundedness of all $\int_{E}\left|f_{\sigma}\right| d \mu$ with $\sigma \in \Delta$. Therefore we have

5.1. A semimartingale $\Phi$ is of bounded variation if and only if the sequence $\left(\widehat{\phi}_{\sigma}\right)$ given by (5.2) is terminally uniformly bounded.

The same theorem is true, of course, with the corresponding notions "from above" or "from below."

A semimartingale $\Phi$ is called absolutely continuous if given any positive number $\epsilon$ there exist a terminal set $\Delta$ in $\Theta$, an element $H$ of $\mathfrak{B}$ with $\mu(H)$ $<+\infty$, and a positive number $\delta$ such that $\sigma \in \Delta, A \in \mathfrak{B}_{\sigma}$, and $\mu(H A)<\delta$ imply $\left|\phi_{\sigma}(A)\right|<\epsilon$. Likewise we define absolute continuity from above or from below. Again we may choose $\Delta=\Theta$ if $\Phi$ is a martingale or in the definition of absolute continuity from above. Furthermore given any Boolean sigmasubalgebra $\mathfrak{A}$ of $\mathfrak{B}$ such that the restriction of $\mu$ to $\mathfrak{A}$ is sigma-finite we can always determine $H$ as an element of $\mathfrak{A}$, in particular as an element of an arbitrarily prescribed $\mathfrak{B}_{\eta}$.

5.2. If the sequence $\left(\widehat{\phi}_{\sigma}\right)$ given by (5.2) is terminally uniformly absolutely continuous, $\Phi$ becomes absolutely continuous.

This statement and the analogous ones with "from above" and "from below" are trivial, and their converses are wrong. However, we have (cf. [4, pp. 344-345] and [8, p. 241] for special cases):

5.3. A semimartingale is of bounded variation and absolutely continuous if and only if it is terminally uniformly integrable, and likewise with the corresponding notions "from above" and "from below."

Proof. The part. "if" follows immediately from 3.1, 5.1, and 5.2. To prove 
the part "only if" let the semimartingale $\Phi$ be of bounded variation and absolutely continuous. Given any positive $\epsilon$ there are an index $\eta$, finite positive numbers $\beta$ and $\delta$, and an element $H$ in $\mathfrak{B}_{\eta}$ of finite measure such that $\eta \ll \sigma$ implies

$$
\int_{E}\left|f_{\sigma}\right| d \mu \leqq \beta
$$

and $\eta \ll \sigma, A \in \mathfrak{B}_{\sigma}$, and $\mu(H A)<\delta$ imply

$$
\int_{A}\left|f_{0}\right| d \mu<\epsilon
$$

With $G_{\sigma}=\left\{\left|f_{\sigma}\right|>\gamma\right\}$ we obtain from (5.3) the inequality

$$
\gamma \mu\left(G_{\sigma}\right) \leqq \int_{G_{\sigma}}\left|f_{\sigma}\right| d \mu \leqq \beta<+\infty
$$

for every $\gamma$ and every $\sigma$ with $\eta \ll \sigma$. Therefore there exists a $\gamma$ such that $\mu\left(G_{\sigma}\right)<\delta$ for every $\sigma$ with $\eta \ll \sigma$, hence $\int_{G_{\sigma}}\left|f_{\sigma}\right| d \mu<\epsilon$, by (5.4), because $G_{\sigma} \in \mathfrak{B}_{\sigma}$. Furthermore the element $G=E-H$ belongs to $\mathfrak{B}_{\sigma}$ with $\mu(H G)=0$ if $\eta \ll \sigma$, and thus from (5.4) follows $\int_{G}\left|f_{\sigma}\right| d \mu<\epsilon$ which proves the terminally uniform integrability of $\left(f_{\sigma}\right)$.

5.4. Suppose that the restriction of $\mu$ to the Boolean sigma-algebra $\bigcap_{\sigma \in \Theta} \mathfrak{B}_{\sigma}$ is sigma-finite. Then every terminally uniformly integrable martingale is uniformly integrable, and likewise with "from above" and "from below." If a semimartingale is terminally uniformly integrable from above, it is also uniformly integrable from above.

Proof. If a martingale $\Phi$ is terminally uniformly integrable, it is of bounded variation and absolutely continuous. The proof of 5.3 then shows that $\Phi$ is even uniformly integrable: (5.3) and (5.4) hold by the semimartingale property of $\left|f_{\sigma}\right|$ for every $\sigma$, and if we choose $H$ in $\bigcap_{\sigma} \mathfrak{B}_{\sigma}$, we have $H \in \mathfrak{B}_{\sigma}$ for every $\sigma$.

From 4.1, 5.3, and the definition of "bounded variation" and "absolutely continuous" follows

5.5. Let $\Phi$ be a semimartingale whose integral exists. If $\Phi$ is of bounded variation or absolutely continuous or terminally uniformly integrable $\tilde{\Phi}$ has the same property, and likewise with the corresponding notions "from above" and "from below." A sufficient but not a necessary condition in order that $\tilde{\Phi}$ exists is that $\Phi$ is of bounded variation from below.

Let $\infty$ denote an element not belonging to $\Theta$, and $\Theta_{\infty}=\Theta \cup\{\infty\}$. We extend the relation $\ll$ from $\Theta$ to $\Theta_{\infty}$ by " $\sigma \ll \infty$ for every $\sigma$ in $\Theta_{\infty}$," and thus obtain again a directed set. Let $\mathfrak{B}_{\infty}$ be any Boolean sigma-subalgebra of $\mathfrak{B}$ containing every $\mathfrak{B}_{o}$. Then 5.3 yields (cf. $[4$, p. 311$]$ ):

5.6. Let $\left(f_{\sigma} ; \sigma \in \Theta_{\infty}\right)$ be a semimartingale relative to $\left(\mathfrak{B}_{\sigma} ; \sigma \in \Theta_{\infty}\right)$ in its inte- 
grand representation, and $\int_{E} f_{\infty} d \mu<+\infty$. Then the semimartingale $\left(f_{\sigma} ; \sigma \in \Theta\right)$ is terminally uniformly integrable from above.

5.7. Let $\left(f_{\sigma} ; \sigma \in \Theta_{\infty}\right)$ be a martingale relative to $\left(\mathfrak{B}_{\sigma} ; \sigma \in \Theta_{\infty}\right)$ in its integrand representation, and $\int_{E}\left|f_{\infty}\right| d \mu<+\infty$. Then the martingale $\left(f_{\sigma} ; \sigma \in \Theta\right)$ is terminally uniformly integrable. Likewise $\int_{E} f_{\infty}^{+} d \mu<+\infty$ or $\int_{E} f_{\infty}^{-} d \mu<+\infty$ implies the terminally uniform integrability of this martingale from above or below, respectively.

6. The Vitali condition. A sequence $\left(K_{\sigma} ; \sigma \in \Theta\right)$ in $\mathfrak{B}$ is called a fine covering of an element $A$ of $\mathfrak{B}$ if the following statement is true:

$\mathrm{C}\left(A,\left(K_{\sigma}\right)\right)$. For every index $\sigma$ we have $K_{\sigma} \in \mathfrak{B}_{\sigma}$, and $A \leqq \mathrm{~V}\left\{K_{\sigma} ; \sigma \in \Delta\right\}$ for every terminal subset $\Delta$ of $\Theta$.

The sequence $\left(\mathfrak{B}_{\sigma} ; \sigma \in \Theta\right)$ is said to enjoy Vitali's property if:

$V_{0}$. Given any fine covering $\left(K_{\sigma}\right)$ of an element $A$ of finite measure and any positive number $\epsilon$ there exist a finite set $\left\{\xi_{1}, \cdots, \xi_{r}\right\}$ of indices and disjoint elements $L_{1}, \cdots, L_{r}$ such that $L_{i} \in \mathfrak{B}_{\xi_{i}}, L_{i} \leqq K_{\xi_{i}}(i=1, \cdots, r)$, and $\mu(A$ $\left.-A \mathrm{~V}_{i=1}^{r} L_{i}\right)<\epsilon$.

We can always assume that the $\xi_{i}$ 's belong to a given terminal set $\Delta$ of indices by replacing $\left(K_{\sigma}\right)$ by the following fine covering of $A: K_{\sigma}^{\prime}=K_{\sigma}$ if $\sigma \in \Delta$, and $K_{\sigma}^{\prime}=O$ if $\sigma \notin \Delta$. It would also be sufficient to state $V_{0}$ only for every $A$ of the least Boolean sigma-subalgebra $\mathfrak{B}_{\infty}$ of $\mathfrak{B}$ containing all $\mathfrak{B}_{\sigma}$ 's because every fine covering of an element $A$ of $\mathfrak{B}$ is a fine covering of the $\mathfrak{B}_{\infty}$-cover of $A$.

$\boldsymbol{C}\left(A,\left(K_{\sigma}\right)\right)$ and $\boldsymbol{V}_{0}$ obviously depend on the relation $\ll$ in $\Theta$, and $\boldsymbol{C}(A$, $\left.\left(K_{\sigma}\right)\right)$ is independent of $\mu$. However, we also have

6.1. Let $\mu^{\prime}$ be another sigma-finite and strictly positive measure on $\mathfrak{B}$. Then if $\left(\mathfrak{B}_{\sigma}\right)$ satisfies $V_{0}$ with the measure $\mu$, it has the same property relative to $\mu^{\prime}$, i.e. $V_{0}$ does not depend on the measure.

Proof. Let $\left(K_{\sigma}\right)$ be a fine covering of $A, \mu^{\prime}(A)<+\infty$, and $\epsilon>0$. Denoting by $h$ a Radon-Nikodým integrand of $\mu^{\prime}$ relative to $\mu$ we have

$$
\mu^{\prime}(G)=\int_{G} h d \mu
$$

for every $G$ in $\mathscr{B}$. Since $\mu^{\prime}(A)<+\infty, h$ is finite in $A$, and therefore there exists an element $B$ in $B$ such that $B \leqq A, \mu^{\prime}(A-B)<\epsilon$, and $h$ is bounded in $B$, say

$$
h<\gamma[B], \quad \text { where } 0<\gamma<+\infty .
$$

Furthermore since $\mu$ is sigma-finite and $\mu^{\prime}(B)$ finite, there exists an element $C$ in $\mathfrak{B}$ such that $C \leqq B, \mu^{\prime}(B-C)<\epsilon$, and $\mu(C)<+\infty$. The sequence $\left(K_{\sigma}\right)$ is also a fine covering of $C$, and applying $V_{0}$ with the measure $\mu$ to $C$ we obtain indices $\xi_{1}, \cdots, \xi_{r}$ and disjoint elements $L_{1}, \cdots, L_{r}$ such that $L_{i} \in \mathfrak{B}_{\xi_{i}}$, $L_{i} \leqq K_{\xi_{i}}$, and $\mu\left(C-C \mathrm{~V}_{i=1}^{r} L_{i}\right)<\epsilon / \gamma$. Thus according to (6.1) and (6.2): $\mu^{\prime}\left(C-C \bigvee_{i=1}^{r} L_{i}\right)<\epsilon$, hence $\mu^{\prime}\left(A-A \bigvee_{i=1}^{r} L_{i}\right)<3 \epsilon$. 
6.2. The Vitali condition $V_{0}$ is satisfied by every sequence $\left(\mathfrak{B}_{\sigma}\right)$ such that the family of all $\mathfrak{B}_{\sigma}$ is totally ordered by $\subseteq$, as e.g. in the case that $\Theta$ is totally ordered by $\ll$.

Proof. Let $\left(K_{\sigma}\right)$ be a fine covering of $A, \mu(A)<+\infty$, and $\epsilon>0$. We have $A \leqq \mathrm{~V}\left\{K_{\sigma} ; \sigma \in \Theta\right\}$ and therefore a Fréchet sequence $\left(\xi_{i} ; i=1,2, \cdots\right)$ such that $A \leqq \bigvee\left\{K_{\xi_{i}} ; i=1,2, \cdots\right\}$. Since $\mu(A)<+\infty$, there exists an integer $r$ with $\mu\left(A-A \mathrm{~V}_{i=1}^{r} K_{\xi_{i}}\right)<\epsilon$. We can, of course, assume that $B_{\xi_{1}} \subseteq B_{\xi_{2}} \subseteq \ldots$ $\subseteq B_{\xi r}$. Then the elements $L_{1}=K_{\xi_{1}}, L_{2}=K_{\xi_{2}}-K_{\xi_{2}} K_{\xi_{1}}, L_{3}=K_{\xi_{3}}-K_{\xi_{3}}\left(K_{\xi_{1}} \bigvee K_{\xi_{2}}\right)$, $\cdots, L_{r}=K_{\xi_{r}}-K_{\xi_{r}}\left(K_{\xi_{1}} \vee \cdots \vee K_{\xi_{r-1}}\right)$ have the required properties.

7. The density theorem. From now on we assume, as already done in $\$ 5$, that the restriction of $\mu$ to every $\mathscr{B}_{\sigma}$ is sigma-finite. We denote the least Boolean sigma-subalgebra of $\mathfrak{B}$ containing each $\mathfrak{B}_{\sigma}$ by $\mathfrak{B}_{\infty}$. The restriction of $\mu$ to $\mathfrak{B}_{\infty}$ is, of course, sigma-finite, too.

7.1. Let $A \in \mathfrak{B}_{\infty}$, and define $\phi_{\sigma}(K)=\mu(A K)$, if $K \in \mathfrak{B}_{\sigma}$. Let $h_{\sigma}$ be the RadonNikodym integrand of $\phi_{\sigma}$ relative to the restriction of $\mu$ to $\mathfrak{B}_{o}$. Then if the sequence $\left(\mathfrak{B}_{\sigma}\right)$ satisfies $V_{0}, \lim _{\sigma} h_{\sigma}$ exists, and is equal to the characteristic function $\chi_{A}$ of $A$.

Proof. Taking any index $\eta$ in $\Theta$ and using that $\mu$ is sigma-finite on $\mathfrak{B}_{\eta}$ we can find a countable disjoint subset $\mathfrak{V}$ of $\mathfrak{B}_{\eta}$ containing only elements of finite measure and such that $\mathrm{V} \mathfrak{Y}=E$. If $Y \in \mathfrak{Y}$ and $\eta \ll \sigma$, we have $Y \in \mathfrak{B}_{\sigma}$ and $Y A \in \mathfrak{B}_{\infty}$, and $h_{\sigma} \chi_{Y}$ is the Radon-Nikodým integrand relative to $\mu$ of $\mu(A Y K)$ as a function of $K$ in $\mathfrak{B}_{\sigma}$. Supposing $\lim _{\sigma} h_{\sigma} \chi_{Y}=\chi_{Y A}$ for every $Y$ in $\mathfrak{Y}$ we obtain our assertion $\lim _{\sigma} h_{\sigma}=\chi_{A}$. Therefore we can from now on assume that $A \leqq Y$, where $Y \in \mathfrak{B}_{\eta}$ and $0<\mu(Y)<+\infty$.

Under this assumption every $h_{\sigma}$ vanishes outside $Y$ if $\eta \ll \sigma$, and since $0 \leqq h_{\sigma} \leqq 1$, these $h_{\sigma}$ and $\lim \sup _{\sigma} h_{\sigma}$ and $\lim \inf _{\sigma} h_{\sigma}$ are summable. The limit $\lim _{\sigma} \int_{E} h_{\sigma} d \mu=\mu(A)$ exists and is finite. Therefore, according to 3.7, in order to prove the existence of $\lim _{\sigma} h_{\sigma}$, it is sufficient to show that both sequences $\left(h_{\sigma}\right)$ and $\left(-h_{\sigma}\right)$ satisfy $F^{+}$. We shall here verify $F^{+}$for $\left(h_{\sigma}\right)$; but we shall only use that

$$
-1 \leqq h_{\sigma} \leqq 1,
$$

that each $h_{\sigma}$ with $\eta \ll \sigma$ vanishes outside $Y$, and the martingale property $\int_{K} h_{\tau} d \mu=\mu(A K)=\int_{K} h_{\sigma} d \mu$ if $\tau \ll \sigma$ and $K \in \mathfrak{B}_{\tau}$, so that the same proof applies to $\left(-h_{\sigma}\right)$.

Let $g_{\tau}=V_{\tau \varepsilon_{\sigma}} h_{\sigma}$. Given any $\rho$ in $\Theta$ and any positive $\epsilon$ define

$$
\gamma=\frac{\epsilon}{\mu(Y)}
$$

and $G_{\tau}=Y\left\{g_{\tau} \leqq \lim \sup _{\sigma} h_{\sigma}+\gamma\right\}$. The sequence $\left(G_{\tau}\right)$ increases monotonely with $\lim _{\tau} G_{\tau}=Y$ because $\lim \sup _{\sigma} h_{\sigma}$ is finite. Thus $\lim _{\tau} \mu\left(Y-G_{\tau}\right)=0$, i.e. there exists an index $\zeta$ such that $G=G_{\zeta}$ satisfies the inequality

$$
\mu(Y-G)<\epsilon .
$$


Of course we can choose $\zeta$ such that also $\eta \ll \zeta$ and $\rho \ll \zeta$. The definition of $G$ yields

$$
g_{5} \leqq \lim _{\sigma} \sup h_{\sigma}+\gamma[G] .
$$

Define $J_{\sigma}=G\left\{h_{\sigma}>\lim \sup _{\sigma} h_{\sigma}-\gamma\right\}$. By (7.4) we have

$$
h_{\sigma} \geqq g_{\zeta}-2 \gamma\left[J_{\sigma}\right] \text {. }
$$

Let $K_{\sigma}$ be the $\mathfrak{B}_{\sigma}$-cover of $J_{\sigma}$. Since $J_{\sigma} \leqq G \leqq Y$ and $Y \in \mathfrak{B}_{\eta}$, we conclude $K_{\boldsymbol{\sigma}} \leqq Y$ for $\eta \ll \sigma$. The finiteness of $\lim \sup _{\sigma} h_{\sigma}$ and the definition of $J_{\sigma}$ imply $\boldsymbol{C}\left(G,\left(K_{\sigma}\right)\right)$. Therefore given any $p$ indices $\sigma_{1}, \cdots, \sigma_{p}$ with $\zeta \ll \sigma_{k}$ according to $V_{0}$ there exist indices $\xi_{1}, \cdots, \xi_{r}$ and disjoint elements $L_{1}, \cdots, L_{r}$ such that $\sigma_{k} \ll \xi_{i}, L_{i} \in \mathfrak{B}_{\xi_{i}}, L_{i} \leqq K_{\xi_{i}}(k=1, \cdots, p ; i=1, \cdots, r)$, and $\mu(G-G L)<\epsilon$, where $L=\mathrm{V}_{i=1}^{r} L_{i}$. Since $\eta \ll \zeta \ll \xi_{i}$, we have $L_{i} \leqq K_{\xi_{i}} \leqq Y$, hence by (7.3):

$$
\mu(Y-L)<2 \epsilon \text {. }
$$

Let $g=\bigvee_{k=1}^{p} h_{\sigma k}$. The definition of $g_{\zeta}$ and $\zeta \ll \sigma_{k}(k=1, \cdots, p)$ imply $g_{\zeta} \geqq g$, and thus by (7.5), for $i=1, \cdots, r: h_{\xi_{i}} \geqq g-2 \gamma\left[J_{\xi_{i}}\right]$. Because of $\sigma_{k} \ll \xi_{i}(k=1, \cdots, p)$ both members of this inequality are $\mathfrak{B}_{\xi_{i}}$-measurable, hence $h_{\xi_{i}} \geqq g-2 \gamma\left[K_{\xi_{i}}\right]$, and so much more $h_{\xi_{i}} \geqq g-2 \gamma\left[L_{i}\right]$. By integrating and using $L_{i} \in \mathscr{B}_{\xi_{i}}$ and (7.2) we obtain

$$
\int_{L_{\boldsymbol{i}}} h_{\xi_{i}} d \mu \geqq \int_{L_{\boldsymbol{i}}} g d \mu-2 \epsilon \frac{\mu\left(L_{i}\right)}{\mu(Y)} .
$$

Choosing now any index $\xi$ such that $\xi_{i} \ll \xi(i=1, \cdots, r)$ we deduce from the martingale property of $\left(h_{\sigma}\right)$ the equation $\int_{L_{i}} f_{\xi_{i}} d \mu=\int_{L_{i}} f_{\xi} d \mu$, and thus, since the elements $L_{i}$ are disjoint with $\bigvee_{i=1}^{r} L_{i}=L$, and $\mu(L) \leqq \mu(Y)$,

$$
\int_{L} h_{\xi} d \mu \geqq \int_{L} g d \mu-2 \epsilon \text {. }
$$

By (7.1), (7.6), and because $h_{\xi}, h_{\sigma_{1}}, \cdots, h_{\sigma_{p}}$ vanish outside $Y$, we have

$$
-2 \epsilon \leqq \int_{E-L} h_{\xi} d \mu, \quad \int_{E-L} g d \mu \leqq 2 \epsilon,
$$

hence by (7.7):

$$
\int_{E} h_{\xi} d \mu \geqq \int_{E} g d \mu-6 \epsilon .
$$

This completes the proof of the existence of $h=\lim _{\sigma} h_{\sigma}$.

Since $0 \leqq h_{\sigma} \leqq 1$, and every $h_{\sigma}$ with $\eta \ll \sigma$ vanishes outside $Y$, the Theorem 3.2 asserts that $\int_{B} h d \mu=\lim _{\sigma} \int_{B} h_{\sigma} d \mu$ for every element $B$ of $\mathfrak{B}$. If in particular $B \in \mathfrak{B}_{\tau}$ for some $\tau, \tau \ll \sigma$ implies $\int_{B} h_{\sigma} d \mu=\mu(A B)$, and therefore $\int_{B} h d \mu=\mu(A B)$. 
As both members of this equation are countably additive functions of $B$, it must also hold if $B \in \mathfrak{B}_{\infty}$, and thus $h=\chi_{A}$.

7.2. Let $\left(K_{\sigma}\right)$ be a fine covering of an element $A$ in $\mathfrak{B}_{\infty}$, unequal to $O$, and $\epsilon a$ positive number. There exists an index $\rho$ and an element $L$ in $\mathfrak{B}_{\rho}$ such that $L \leqq K_{\rho}, \mu(L)<+\infty$, and $\mu(L A)>(1-\epsilon) \mu(L)$.

Proof. Otherwise we would have, for every index $\tau$, every $\sigma$ with $\tau \ll \sigma$, and every $L$ in $\mathfrak{B}_{\sigma}$ with $O<L \leqq K_{\sigma}$ and $\mu(L)<+\infty$ :

$$
\mu(L A) / \mu(L) \leqq 1-\epsilon .
$$

This would imply $h_{\sigma} \leqq 1-\epsilon\left[K_{\sigma}\right]$ and therefore $\Lambda_{\tau_{\varepsilon \sigma}} h_{\sigma} \leqq 1-\epsilon\left[K_{\sigma}\right]$, hence $\Lambda_{\tau^{*} \sigma} h_{\sigma} \leqq 1-\epsilon[A]$, because $A \leqq \bigvee_{\tau \ll \sigma} K_{\sigma}$. So we would have

$$
\lim _{\sigma} \inf h_{\sigma} \leqq 1-\epsilon[A]
$$

which would contradict $A \neq O$ and $\lim _{\sigma} h_{\sigma}=\chi_{A}$.

8. The martingale convergence theorem. Again we suppose that $\mu$ has a sigma-finite restriction to every $\mathscr{B}_{\sigma}$. We shall deal with the integrand representation $\left(f_{\sigma}\right)$ of a martingale; then convergence of this martingale means, of course, convergence of the sequence $\left(f_{\sigma}\right)$ as defined in $\$ 3$.

8.1. If the sequence $\left(\mathfrak{B}_{\sigma}\right)$ satisfies Vitali's condition $V_{0}$, every martingale $\left(f_{\sigma}\right)$ of bounded variation from above or from below converges, and its limit is integrable with $\int_{E}\left(\lim _{\sigma} f_{\sigma}\right) d \mu<+\infty$ or $-\infty<\int_{E}\left(\lim _{\sigma} f_{\sigma}\right) d \mu$, respectively.

Proof. According to 4.4, (4.7) we may assume that $0 \leqq f_{\sigma}$ for every $\sigma$. If $\left(f_{\sigma}\right)$ were not convergent, there would exist two positive finite numbers $\alpha$ and $\beta$ such that $\alpha<\beta$ and $A=\left\{\lim _{\inf } f_{\sigma}<\alpha\right\}\left\{\beta<\lim \sup _{\sigma} f_{\sigma}\right\} \neq O$. The functions $\lim \inf _{\sigma} f_{\sigma}$ and $\lim \sup _{\sigma} f_{\sigma}$ are measurable relative to the least Boolean sigmaalgebra $\mathfrak{B}_{\infty}$ containing every $\mathfrak{B}_{\sigma}$, and therefore $A \in \mathfrak{B}_{\infty}$. The sequence $K_{\sigma}$ $=\left\{f_{\sigma} \leqq \alpha\right\}$ is a fine covering of $A$. Hence by 7.2, given any number $\epsilon$ with $0<\epsilon<1$, there exist an index $\rho$ and an element $L$ in $\mathfrak{B}_{\rho}$ such that $L \leqq K_{\rho}$, $\mu(L)<+\infty$, and

$$
\dot{\mu}(L A)>(1-\epsilon) \mu(L) .
$$

In particular we have

$$
f_{\rho} \leqq \alpha[L] .
$$

Define now $J_{\sigma}=L\left\{f_{\sigma} \geqq \beta\right\}$ if $\rho \ll \sigma$, and otherwise $J_{\sigma}=E$. Then $J_{\sigma} \in \mathfrak{B}_{\sigma}$ in any case because $L \in \mathfrak{B}_{\sigma}$ for $\rho \ll \sigma$. Therefore $\left(J_{\sigma}\right)$ is a fine covering of $L A$. Since $\mu(L A) \leqq \mu(L)<+\infty$, from $V_{0}$ follows the existence of indices $\xi_{i}$ and disjoint elements $L_{i}$ such that $\rho \ll \xi_{i}, L_{i} \in \mathfrak{B}_{\xi_{i}}, L_{i} \leqq J_{\xi_{i}}(i=1, \cdots, r)$, and $\mu(L A$ $\left.-L A L^{\prime}\right)<\epsilon \mu(A L)$, where $L^{\prime}=\mathrm{V}_{i=1}^{r} L_{i}$. Hence by $(8.1): \mu\left(L^{\prime}\right) \geqq(1-\epsilon) \mu(L A)$ $>(1-\epsilon)^{2} \mu(L)$. This together with $f_{\xi_{i}} \geqq \beta\left[J_{\xi_{i}}\right]$ and $\beta>0$ implies

$$
\sum_{i} \int_{L_{i}} f_{\xi_{i}} d \mu \geqq \beta \mu\left(L^{\prime}\right)>\beta(1-\epsilon)^{2} \mu(L) \text {. }
$$


Choosing any index $\xi$ with $\xi_{i} \ll \xi(i=1, \cdots, r)$ we obtain from the martingale property of $\left(f_{\sigma}\right): \int_{L_{i}} f_{\xi_{i}} d \mu=\int_{L_{i}} f_{\xi} d \mu(i=1, \cdots, r)$, and thus, since $f_{\xi} \geqq 0$ :

$$
\int_{L} f_{\xi} d \mu \geqq \int_{L^{\prime}} f_{\xi} d \mu=\sum_{i} \int_{L_{i}} f_{\xi} d \mu=\sum_{i} \int_{L_{i}} f_{\xi_{i}} d \mu>\beta(1-\epsilon)^{2} \mu(L) .
$$

On the other hand by (8.2), $\rho \ll \xi$, and $L \in \mathfrak{B}_{\rho}$ we have $\int_{L} f_{\xi} d \mu=\int_{L} f_{\rho} d \mu \leqq \alpha \mu(L)$, hence $\alpha \mu(L)>\beta(1-\epsilon)^{2} \mu(L)$, i.e. $\alpha>\beta(1-\epsilon)^{2}$ which contradicts $\alpha<\beta$ if $\epsilon$ is sufficiently small.

The remaining part of the proof follows from 3.4 and 5.1.

Let $\Theta_{\infty}$ denote again the index set obtained from $\Theta$ by adding a "last" element, and $\mathfrak{B}_{\infty}$ the least Boolean sigma-subalgebra of $\mathfrak{B}$ containing every $\mathfrak{B}_{\sigma}$.

8.2. Suppose the sequence $\left(\mathfrak{B}_{\sigma}\right)$ satisfies Vitali's condition $V_{0}$, and $\left(f_{\sigma}\right)$ is a martingale. Then the following conditions are equivalent:

I. The martingale $\left(f_{\sigma} ; \sigma \in \Theta\right)$ is terminally uniformly integrable from above.

II. The martingale $\left(f_{\sigma} ; \sigma \in \Theta\right)$ converges to a function $f_{\infty}$ with $\int_{E} f_{\infty}^{+} d \mu<+\infty$, and $\lim _{\sigma} \int_{E}\left|f_{\infty}^{+}-f_{\sigma}^{+}\right| d \mu=0$.

III. The martingale $\left(f_{\sigma} ; \sigma \in \Theta\right)$ converges to a function $f_{\infty}$, and $\lim _{\sigma} \int_{E} f_{\sigma}^{+} d \mu$ $=\int_{E} f_{\infty}^{+} d \mu<+\infty$.

IV. The martingale $\left(f_{\sigma} ; \sigma \in \Theta\right)$ converges to an integrable function $f_{\infty}$, and $\lim _{\sigma} \int_{A} f_{\sigma} d \mu \leqq \int_{A} f_{\infty} d \mu<+\infty$ for every $A$ in $\bigcup_{\sigma} \mathfrak{B}_{\sigma}$.

V. The martingale $\left(f_{\sigma} ; \sigma \in \Theta\right)$ converges to an integrable function $f_{\infty}$ with $\int_{E} f_{\infty} d \mu<+\infty$, and $\left(f_{\sigma} ; \sigma \in \Theta_{\infty}\right)$ is a semimartingale.

Proof. The condition II follows from I by 3.2, 5.3, and 8.1, and III is a trivial consequence of II. The condition III implies IV by (3.3) and 3.3, and V means obviously the same as IV. Finally I follows from V by 5.6.

Analogous conditions exist, of course, in order that a martingale is terminally uniformly integrable from below. Finally using $3.2,3.5,5.3,5.7$, and 8.1 we obtain

8.3. Suppose the sequence $\left(\mathfrak{B}_{\sigma}\right)$ satisfies Vitali's condition $V_{0}$, and $\left(f_{\sigma}\right)$ is a martingale. Then the following conditions are equivalent:

I. The martingale $\left(f_{\sigma} ; \sigma \in \Theta\right)$ is terminally uniformly integrable.

II. The martingale $\left(f_{\sigma} ; \sigma \in \Theta\right)$ converges to a summable function $f_{\infty}$, and $\lim _{\sigma} \int_{E}\left|f_{\infty}-f_{\sigma}\right| d \mu=0$.

III. The martingale $\left(f_{\sigma} ; \sigma \in \Theta\right)$ converges to a summable function $f_{\infty}$, and $\lim _{\sigma} \int_{E}\left|f_{\sigma}\right| d \mu=\int_{E}\left|f_{\infty}\right| d \mu$.

IV. The martingale $\left(f_{\sigma} ; \sigma \in \Theta\right)$ converges to a summable function $f_{\infty}$, and $\lim _{\sigma} \int_{A} f_{\sigma} d \mu=\int_{A} f_{\infty} d_{\mu}$ for every $A$ in $\bigcup_{\sigma} \mathfrak{B}_{\sigma}$.

V. The martingale $\left(f_{\sigma} ; \sigma \in \Theta\right)$ converges to a summable function $f_{\infty}$, and $\left(f_{\sigma} ; \sigma \in \Theta_{\infty}\right)$ is a martingale.

By 5.4 we can omit the adverb "terminally" in 8.2 , I and 8.3 , I if the restriction of $\mu$ to $\bigcap_{\sigma} \mathfrak{B}_{\sigma}$ is sigma-finite. 
As to the conditions 8.2 , III and 8.3 , III it should be noted that by (3.3) we have

$$
\begin{aligned}
\int_{A} f_{\infty}^{+} d \mu \leqq & \lim _{\sigma} \int_{A} f_{\sigma}^{+} d \mu, \quad \int_{A} \overline{f_{\infty}} d \mu \leqq \lim \int_{A^{\prime}} \overline{f_{\sigma}} d \mu, \\
& \int_{A}\left|f_{\infty}\right| d \mu \leqq \lim _{\sigma} \int_{A}\left|f_{\sigma}\right| d \mu
\end{aligned}
$$

for every martingale $\left(f_{\sigma}\right)$ convergent to $f_{\infty}$ and every $A$ in $U_{\sigma} \mathfrak{B}_{\sigma}$.

The second part of the condition $8.3, \mathrm{~V}$ is, of course, equivalent to the fact that the indefinite integral $\phi_{\sigma}$ of $f_{\infty}$ on $\mathfrak{B}_{\sigma}$ is the restriction to $\mathfrak{B}_{\sigma}$ of the indefinite integral $\phi_{\infty}$ of $f_{\infty}$ on $\mathfrak{B}_{\infty}$, and that $\phi_{\infty}$ remains finite. In some cases a $\mathfrak{B}_{\infty}$-measurable and summable function $f_{\infty}$ only is given primarily, or, what amounts to the same thing, its indefinite integral on $\mathfrak{B}_{\infty}$, i.e. a finite countably additive function $\phi_{\infty}$ on $\mathfrak{B}_{\infty}$. Denoting by $\phi_{\sigma}$ the restriction of $\phi_{\infty}$ to $\mathfrak{B}_{\sigma}$ and by $f_{\sigma}$ the Radon-Nikodým integrand of $\phi_{\sigma}$ relative to the restriction of $\mu$ to $\mathfrak{B}_{\sigma}$ we obtain the integral- and integrand representation of a martingale relative to $\left(\mathfrak{B}_{\sigma} ; \sigma \in \Theta\right)$ which is terminally uniformly integrable by 5.7 . Hence $f_{\infty}=\lim _{\sigma} f_{\sigma}$ by 8.3 if $\left(\mathfrak{B}_{\sigma} ; \sigma \in \Theta\right)$ satisfies the Vitali condition. This statement is a generalization of the Density Theorem 7.1 in which $\phi_{\infty}(K)=\mu(A K)$.

9. A limit theorem on semimartingales. The same assumptions and notations as in $\S 8$ are valid.

9.1. Suppose the sequence $\left(\mathscr{B}_{\sigma} ; \sigma \in \Theta\right)$ satisfies Vitali's condition $V_{0}$. Then if the semimartingale $\Phi=\left(f_{\sigma}\right)$ is terminally uniformly integrable from above, $\lim \sup _{\sigma} f_{\sigma}$ becomes integrable, and

$$
\lim _{\sigma} \int_{A} f_{\sigma} d \mu \leqq \int_{A}\left(\limsup _{\sigma} f_{\sigma}\right) d \mu<+\infty
$$

for every $A$ in $\cup_{\sigma} \mathfrak{B}_{\sigma}$. If $\left(f_{\sigma}\right)$ is terminally uniformly integrable, lim $\sup _{\sigma} f_{\sigma}$ becomes summable, and

$$
\lim _{\sigma} \int_{A} f_{\sigma} d \mu=\int_{A}\left(\limsup _{\sigma} f_{\sigma}\right) d \mu
$$

for every $A$ in $\cup_{\sigma} \mathfrak{B}_{\sigma}$.

Proof. The sequence $\left(f_{\sigma}^{+}\right)=\sup (\Phi, 0)$ is also a semimartingale, and its integral $\int \sup (\Phi, 0)$ exists and is terminally uniformly integrable by 5.5 . Let $\left(\tilde{f}_{\sigma}^{+}\right)$be an integrand representation of $\int \sup (\Phi, 0)$. By 8.2 the sequence $\left(\tilde{f}_{\sigma}^{+}\right)$converges to a summable limit, and we have $\lim \sup _{\sigma} f_{\sigma} \leqq \lim \sup _{\sigma} f_{\sigma}^{+}$ $\leqq \lim _{\sigma} \tilde{f}_{\sigma}^{+}$. Hence $\lim \sup _{\sigma} f_{\sigma}$ is integrable with $\int_{E}\left(\lim \sup _{\sigma} f_{\sigma}\right) d \mu<+\infty$, and this together with 3.2 implies (9.1) if $A \in \mathrm{U}_{\sigma} \mathfrak{B}_{\sigma}$.

If $\left(f_{\sigma}\right)$ is terminally uniformly integrable, its integral $\tilde{\Phi}$ exists, and is terminally uniformly integrable by 5.5 . Let $\left(\tilde{f}_{\sigma}\right)$ be an integrand representa- 
tion of $\tilde{\Phi}$. Then $\lim _{\sigma} \tilde{f}_{\sigma}$ exists and is summable by 8.3 , and from lim $\sup _{\sigma} f_{\sigma}$ $\leqq \lim _{\sigma} \tilde{f}_{\sigma}$, from 8.3 , and the definition of $\widetilde{\Phi}$ we obtain

$$
\int_{A}\left(\limsup _{\sigma} f_{\sigma}\right) d \mu \leqq \int_{A}\left(\lim _{\sigma} \bar{f}_{\sigma}\right) d \mu=\lim _{\sigma} \int_{\boldsymbol{A}} \tilde{f}_{\sigma} d \mu=\lim _{\sigma} \int_{\boldsymbol{A}} f_{\sigma} d \mu
$$

if $A \in \cup_{\sigma} \mathfrak{B}_{\sigma}$. Therefore by (9.1):

$$
\int_{A}\left(\limsup f_{\sigma}\right) d \mu=\int_{A}\left(\lim _{\sigma} \tilde{f}_{\sigma}\right) d \mu=\lim _{\sigma} \int_{A} f_{\sigma} d \mu .
$$

This completes the proof.

The function $f_{\infty}=\lim \sup _{\sigma} f_{\sigma}$ is $\mathfrak{B}_{\infty}$-measurable. If $\left(f_{\sigma}\right)$ is terminally uniformly integrable from above and $\left(\mathfrak{B}_{\sigma}\right)$ satisfies $V_{0}, f_{\infty}$ becomes integrable with $\int_{E} f_{\infty} d \mu<+\infty$, and $(9.1)$ asserts that $\left(f_{\sigma} ; \sigma \in \Theta_{\infty}\right)$ is also a semimartingale. On the other hand the existence of a $\mathfrak{B}_{\infty}$-measurable function $f_{\infty}$ with $\int_{E f_{\infty}} d \mu$ $<+\infty$ and such that $\left(f_{\sigma} ; \sigma \in \Theta_{\infty}\right)$ is a semimartingale implies by 5.6 the terminally uniform integrability of $\left(f_{\sigma} ; \sigma \in \Theta\right)$ from above.

10. Cell functions. By a partition of $E$ we mean a disjoint subset $\sigma$ of $\mathscr{B}$ with $\mathrm{V} \sigma=E$ containing only elements of finite measure which are unequal to $O$. Since $\mu$ is sigma-finite, every partition is countable. Each element of $\sigma$ is called a component of $\sigma$. A partition $\sigma$ is said to be finer than a partition $\tau$, symbolically $\tau \ll \sigma$, if every component of $\sigma$ is a part of some component of $\tau$. The relation 《 is obviously transitive. Let now $\Theta$ be a nonempty set of partitions of $E$ directed by $\ll$. Denoting by $\mathfrak{B}_{\sigma}$ the set of all suprema of subsets of $\sigma$ we obtain a monotone increasing sequence $\left(\mathfrak{B}_{\sigma} ; \sigma \in \Theta\right)$ of Boolean sigmasubalgebras of $\mathfrak{B}$, and the restriction of $\mu$ to every $\mathfrak{B}_{\sigma}$ is sigma-finite.

Each element of $\mathscr{B}$ which is a component of some partition belonging to $\Theta$ is called a cell. Let $\Omega$ denote the system of all cells, and let $\phi$ be a cell function, i.e. a real function on $\Re$ which need not be finite. For every disjoint system $\Im$ of cells let $\widehat{\phi}(\Im)=\sum_{J \in \Im} \phi(J)$ provided that this series converges unconditionally. Any element $A$ of $\mathfrak{B}_{\sigma}$ with $\sigma \in \Theta$ has a unique representation as a supremum of a disjoint subset $\Im$ of $\sigma$, and we define $\phi_{\sigma}(A)=\widehat{\phi}(\Im)$ assuming that $\widehat{\phi}(\Im)$ exists for any such $\Im$. Then $\phi_{\sigma}(K)=\phi(K)$ if $K$ is a component of $\sigma$.

The cell function $\phi$ is called semiadditive if for every disjoint system $\Im$ of cells contained in a partition $\sigma$ whose supremum is a component of a partition $\tau$ with $\tau \ll \sigma$ we have $\phi(\bigvee \Im) \leqq \widehat{\phi}(\Im)$. This is equivalent to the fact that $\left(\phi_{\sigma} ; \sigma \in \Theta\right)$ is a semimartingale relative to $\left(\mathfrak{B}_{\sigma} ; \sigma \in \Theta\right)$. The cell function $\phi$ is called additive if for every disjoint system $\Im$ of cells contained in a partition $\sigma$ whose supremum is a cell we have $\phi(\mathrm{V} \Im)=\widehat{\phi}(\Im)$. It amounts to the same thing to require this equality only in the case that $V \Im$ is a component of some partition $\tau$ with $\tau \ll \sigma$. The cell function $\phi$ is additive if and only if $\left(\phi_{\sigma} ; \sigma \in \Theta\right)$ is a martingale relative to $\left(\mathfrak{B}_{\sigma} ; \sigma \in \Theta\right)$. On the other hand any 
martingale $\left(\phi_{\sigma} ; \sigma \in \Theta\right)$ relative to $\left(\mathfrak{B}_{\sigma} ; \sigma \in \Theta\right)$ arises in this way from a cell function $\phi$. Indeed, if $K$ is a cell, $\phi_{\sigma}(K)$ does not depend on $\sigma$, and thus defines $\phi(K)$. In particular if $\phi$ is semiadditive and $-\infty<\widehat{\phi}(\rho)$ for at least one partition $\rho$ of $\Theta$, the integral of the semimartingale $\left(\phi_{\sigma}\right)$ exists, and induces an additive cell function $\tilde{\phi}$ which will be called the integral of $\phi$. The value $\tilde{\phi}(K)$, where $K \in \Omega$, is obviously the Burkill-Kolmogorov integral $\int_{K} \phi$ of $\phi$ over $K$ relative to the relation « restricted to $K$ as considered in $[8$, p. 238].

Let $\mathfrak{C}$ be a set of semiadditive cell functions. If the supremum as given by (4.3) of the semimartingales generated by the elements of $\mathcal{C}$ exists in the space of all semimartingales, it is also generated by a semiadditive cell function $\psi$, namely $\psi(K)=\mathrm{V}\{\phi(K) ; \phi \in \mathfrak{C}\}$ for every $K$ in $\Omega$. In particular if $\Phi$ is generated by $\phi$, sup $(\Phi, 0)$ is generated by $\phi^{+}$, where $\phi^{+}(K)=(\phi(K))^{+}=\phi(K) \bigvee 0$ for every cell $K$. In the case of an additive cell function, $\sup (-\Phi, 0)$ corresponds to $\phi^{-}$and $\sup (-\Phi, \Phi)$ to $|\phi|$. Therefore $\Phi^{+}$is generated by $\int_{K} \phi^{+}, \Phi^{-}$ by $\int_{K} \phi^{-}$, and $|\Phi|$ by $\int_{K}|\phi|$, these integrals considered as functions of the cell $K$. We call them the positive, negative, and total variation of $\phi$.

A semiadditive cell function is said to be of bounded variation or absolutely continuous if the semimartingale it generates has this property. Likewise the corresponding notions "from above" or "from below" are defined. These are the notions relative to the "fineness by partitions" as considered in $[8, \mathrm{pp}$. 240-241]. However, it is obvious that any additive cell function having one of these properties relative to the fineness by partitions has the same property also "universally" as defined loc. cit., and conversely. The same is true with semiadditive cell functions and the properties "from above."

By 4.4 and 4.5 every additive cell function of bounded variation from above or from below is the difference of its positive and its negative variation, and the space of all additive cell functions of bounded variation is a conditionally complete vector lattice.

Let $\phi$ be a semiadditive cell function, and $\left(\phi_{\sigma}\right)$ the semimartingale it generates. The Radon-Nikodým integrand of $\phi_{0}$ relative to the restriction of $\mu$ to $\mathfrak{B}_{\sigma}$ is the so-called $\sigma$-derivative of $\phi$, i.e. the function $D(\phi, \sigma)$ which is constant in each component $J$ of $\sigma$ with the value $\phi(J) / \mu(J)$. Therefore $\lim \inf _{\sigma} D(\phi, \sigma)$ is the lower and $\lim \sup _{\sigma} D(\phi, \sigma)$ the upper derivative of $\phi$, the convergence of the sequence $(D(\phi, \sigma))$ is equivalent to the differentiability of $\phi$, and $D \phi=\lim _{\sigma} D(\phi, \sigma)$.

A set $\mathfrak{R}$ of cells is called a fine covering of an element $A$ of $\mathfrak{B}$ if the following statement is true:

$\hat{\boldsymbol{C}}(A, \mathfrak{R})$. For every terminal set $\Delta$ in $\Theta$ we have $A \leqq \mathrm{~V}(\mathfrak{R} \cap \cup \Delta)$.

If a set $\mathfrak{R}$ of cells satisfies $\hat{\boldsymbol{C}}(A, \mathfrak{R})$, the sequence $\left(K_{\sigma}\right)$ given by $K_{0}=\mathrm{V}\left(\mathfrak{R} \cap \mathfrak{B}_{\sigma}\right)$ satisfies $\boldsymbol{C}\left(A,\left(K_{\sigma}\right)\right)$. On the other hand if we have $\boldsymbol{C}\left(A,\left(K_{\sigma}\right)\right)$ and if $\Im_{\sigma}$ denotes the subset of $\sigma$ such that $K_{\sigma}=\mathrm{V} \Im_{\sigma}$, then $\mathfrak{R}=\mathrm{U}_{\sigma} \Im_{\sigma}$ satisfies $\mathrm{C}(A, \mathfrak{R})$. Therefore the Vitali condition $V_{0}$ on $\left(\mathfrak{B}_{\sigma}\right)$ becomes equivalent to the following condition on $\Theta$ : 
$V_{0}$. Given any fine covering $\mathfrak{R}$ of an element $A$ in $\mathfrak{B}$ of finite measure by cells and any positive number $\epsilon$ there exists a finite and disjoint subset $\mathfrak{B}$ of $\mathfrak{R}$ with $\mu(A-A \bigvee \mathfrak{P})<\epsilon$.

From 8.1-8.3 we obtain finally

10.1. Suppose that $\Theta$ satisfies Vitali's condition $V_{0}$, and $\phi$ is an additive cell function. If $\phi$ is of bounded variation from above or from below, $\phi$ becomes differentiable with an integrable derivative, and $\int_{E} D \phi d \mu<+\infty$ or $-\infty<\int_{E} D \phi d \mu$ respectively. The cell function $\phi$ is of bounded variation from above and absolutely continuous from above if and only if $\phi$ is differentiable with an integrable derivative, $\int_{E} D \phi d \mu<+\infty ;$ and $\phi(K) \leqq \int_{K} D \phi d \mu$ for every cell $K$. The analogous statement is true with "from below" instead of "from above." The cell function $\phi$ is of bounded variation and absolutely continuous if and only if it is differentiable with a summable derivative, and $\phi(K)=\int_{K} D \phi d \mu$ for every cell $K$.

11. Point functions and separability. In opposition to the preceding chapters we shall now denote by $E$ a nonempty set, by $\mathfrak{B}$ a Boolean sigmasetalgebra of subsets of $E$ containing $E$, by $O$ the empty set, and by $\mu$ a positive sigma-finite measure defined on $\mathfrak{B}$. Let $\mathfrak{R}$ be the sigma-ideal in $\mathfrak{B}$ of all sets $N$ of $\mathfrak{B}$ such that $\mu(N)=0$, and $\mathfrak{R}^{*}$ the system of all subsets of sets of $\mathfrak{N}$. Relations "mod $\mathfrak{N}$ " or "mod $\mathfrak{N}^{*}$ " among sets or real functions on $E$ are defined as usual; e.g. $A \subseteq B$ mod $\mathfrak{N}$ means $A-A B \in \mathfrak{N}$, or $f \leqq g$ mod $\mathfrak{N}$ means $\{x: f(x)>g(x)\} \in \mathfrak{N}$.

The ordinary, pointwise least upper bound and greatest lower bound of a system $(B)$ of real functions on $E$ will be denoted by $V^{*}(S)$ and $\Lambda^{*}(S)$, i.e. $\left(V^{*}(\mathbb{S})(x)=\bigvee_{f \in \Theta} f(x)\right.$ for every $x$ in $E$, and likewise $\Lambda^{*}(S$. Similarly we have a pointwise limit inferior and limit superior of a Moore-Smith sequence $\left(f_{\sigma}\right.$; $\sigma \in \Theta)$ of real functions:

$$
\underset{\sigma}{\liminf } f_{\sigma}=\underset{\tau \in \Theta}{\bigvee^{*}} \underset{\tau \ll \sigma}{\Lambda^{*}} f_{\sigma}, \quad \quad \lim \sup ^{*} f_{\sigma}=\underset{\tau \in \Theta}{\Lambda^{*}} \underset{\tau \in \sigma}{\bigvee^{*}} f_{\sigma}
$$

Every $\mathfrak{B}$-measurable "pointfunction" $f$ on $E$ determines a $\mathfrak{B} / \mathfrak{N}$-measurable function which is essentially the class of all $\mathscr{B}$-measurable functions equal to $f \bmod \mathfrak{N}$. Since $\mu$ induces a sigma-finite strictly positive measure on $\mathfrak{B} / \mathfrak{N}$, the $\mathfrak{B} / \mathfrak{N}$-measurable functions determined by the elements of a set $\mathbb{B}$ of $\mathfrak{B}$-measurable functions have a supremum and an infimum as considered in $\S 2$, and by $V(S)$ and $\Lambda \&$ we mean any representative of these classes. These two functions are $\mathfrak{B}$-measurable, but only determined mod $\mathfrak{N}$, and they are left unaltered if each function in $\leftrightarrow$ is changed in a set of $\mathfrak{R}$. We call them the supremum and infimum of $(S)$ mod $\mathfrak{N}$." Similarly we obtain the limit inferior and superior "mod $\mathfrak{N}$ " of a stochastic process $\left(f_{\sigma} ; \sigma \in \Theta\right)$, i.e. a sequence of $\mathfrak{B}$-measurable functions; we denote them again by $\lim \inf _{\sigma} f_{\sigma}$ and $\lim \sup _{\sigma} f_{\sigma}$. They are also $\mathfrak{B}$-measurable, uniquely determined $\bmod \mathfrak{N}$, and invariant under a change of each $f_{\sigma}$ on a set of $\mathfrak{N}$.

11.1. For every system (S) of $\mathfrak{B}$-measurable functions on $E$ we have $\Lambda^{*}(B)$ $\leqq \Lambda \leftrightarrow \leqq V\left(S \leqq V * G \bmod \mathfrak{R}^{*}\right.$. 
Proof. Let $g$ be the infimum "mod $\mathfrak{R}$ " of all $\mathfrak{B}$-measurable functions $h$

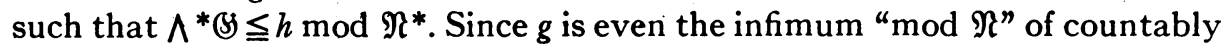
many functions $h$ with this property, we have also $\Lambda^{*}(B) \leqq g \bmod \mathfrak{N}^{*}$. On the other hand $\Lambda^{*}(\mathfrak{S} \leqq f$ holds for every function $f$ of $\mathfrak{G}$, therefore $g \leqq f \bmod \mathfrak{R}$, herice $g \leqq \Lambda \leftrightarrow \bmod \mathfrak{N}$. This implies $\Lambda^{*}\left(\mathfrak{S} \leqq \Lambda \circlearrowleft \mathcal{G o d} \mathfrak{N}^{*}\right.$, and similarly we obtain $V(S) V^{*}(S)$.

From now on we shall always assume that the index set $\Theta$ and the relation $\ll$ in it satisfy the following hypothesis:

A. There exists a countable cofinal subset of $\Theta$.

11.2. Let $\left(f_{\sigma} ; \sigma \in \Theta\right)$ be a stochastic process. Then

$$
\lim _{\sigma} \inf ^{*} f_{\sigma} \leqq \liminf f_{\sigma} \leqq \lim \sup _{\sigma} f_{\sigma} \leqq \lim \sup _{\sigma}^{*} f_{\sigma} \bmod \mathfrak{R}^{*} .
$$

Therefore, if the sequence of numbers $\left(f_{\sigma}(x) ; \sigma \in \Theta\right)$ converges for almost all $x$, the sequence $\left(f_{\sigma} ; \sigma \in \Theta\right)$ converges "mod $\mathfrak{R}$."

Proof. By 11.1 we have $\Lambda_{r \ll \sigma}^{*} f_{\sigma} \leqq \Lambda_{r \ll \sigma} f_{\sigma} \bmod \mathfrak{N}^{*}$. This inequality with every $\tau$ of a countable cofinal subset of $\Theta$ implies $\lim _{\sigma} \inf _{\sigma}{ }^{*} f_{\sigma} \leqq \lim \inf _{\sigma} f_{\sigma} \bmod \mathfrak{N}^{*}$.

11.3. Given any stochastic process $\left(f_{\sigma} ; \sigma \in \Theta\right)$ there exists a countable cofinal set $\Gamma$ in $\Theta$ such that

$$
\liminf _{\sigma \in \boldsymbol{\Theta}} f_{\sigma}=\liminf _{\sigma \in \Gamma} f_{\sigma} \bmod \mathfrak{N}, \quad \quad \limsup _{\sigma \in \boldsymbol{\Theta}} f_{\sigma}=\limsup _{\sigma \in \Gamma} f_{\sigma} \bmod \mathfrak{N} .
$$

Proof. Let $\left\{\Delta_{1}, \Delta_{2}, \cdots\right\}$ be a countable basis of the filter of all terminal sets in $\Theta$. For every $k$ there exists a countable subset $\Gamma_{k}$ of $\Delta_{k}$ such that

$$
\bigwedge_{\sigma \in \Delta_{k}} f_{\sigma}=\bigwedge_{\sigma \in \Gamma_{k}} f_{\sigma} \bmod \mathfrak{N}, \quad \bigvee_{\sigma \in \Delta_{k}} f_{\sigma}=\bigvee_{\sigma \in \Gamma_{k}} f_{\sigma} \bmod \mathfrak{R}
$$

The union $\Gamma=U_{k} \Gamma_{k}$ has the required property.

The stochastic process $\left(f_{\sigma} ; \sigma \in \Theta\right)$ is called separable if 11.3 holds also with the "pointwise" suprema and infima, more precisely if there exists a countable cofinal subset $\Gamma$ of $\Theta$ such that

$$
\liminf _{\sigma \in \Theta} f_{\sigma}=\liminf _{\sigma \in \Gamma} f_{\sigma} \bmod \mathfrak{N}^{*}, \quad \limsup _{\sigma \in \Theta}^{*} f_{\sigma}=\limsup _{\sigma \in \Gamma} f_{\sigma} \bmod \mathfrak{N}^{*} .
$$

(11.1) implies that the functions $\lim \inf _{\sigma \in \Theta}^{*} f_{\sigma}$ and $\lim \sup _{\sigma \in \Theta}^{*} f_{\sigma}$ are measurable relative to the completion of $\mu$.

11.4. A stochastic process $\left(f_{\sigma}\right)$ is separable if and only if

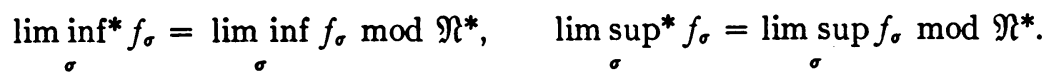

Proof. Let $\left(f_{\sigma}\right)$ be separable, and $\Gamma$ countable and satisfying (11.1). Then

$$
\liminf _{\sigma \in \Theta} f_{\sigma} \leqq \liminf _{\sigma \in \Gamma} f_{\sigma}=\liminf _{\sigma \in \Gamma} f_{\sigma}=\liminf _{\sigma \in \Theta} f_{\sigma} \bmod \mathfrak{N}^{*} .
$$

Hence the first equation (11.2) follows from 11.2. Likewise we obtain the 
second one. On the other hand let (11.2) be true, and let $\Gamma$ be determined as in 11.3. Then

$$
\liminf _{\sigma \in \Theta} f_{\sigma}=\liminf _{\sigma \in \Theta} f_{\sigma}=\liminf _{\sigma \in \Gamma} f_{\sigma}=\liminf _{\sigma \in \Gamma} f_{\sigma} \bmod \Re^{*},
$$

and similarly $\lim \sup _{\sigma \in \Theta}^{*} f_{\sigma}=\lim \sup _{\sigma \in \Gamma}^{*} f_{\sigma} \bmod \mathfrak{R}^{*}$.

The following lemma follows from a more general one proved by Doob $[4$, p. 56].

11.5. Let $\left(f_{\sigma}\right)$ be a stochastic process. There exists a countable subset $\Gamma$ of $\Theta$ and to every index $\sigma$ a set $N_{\sigma}$ in $\mathfrak{\Re}$ such that $x \notin N_{\sigma}$ implies

$$
\bigwedge_{\tau \in \Gamma} f_{\tau}(x) \leqq f_{0}(x) \leqq \bigvee_{\tau \in \Gamma} f_{\tau}(x) .
$$

11.6. Given any stochastic process $\left(f_{\sigma}\right)$ there is a separable stochastic process $\left(f_{\sigma}^{*}\right)$ such that $f_{\sigma}=f_{\sigma}^{*} \bmod \mathfrak{N}$ for every index $\sigma$.

Proof. Let $\left\{\Delta_{1}, \Delta_{2}, \cdots\right\}$ be a countable basis of the filter of all terminal sets in $\Theta$. Applying 11.5 to every process $\left(f_{\sigma} ; \sigma \in \Delta_{k}\right)$ we obtain countable sets $\Gamma_{k}$ of indices and to each $\sigma$ in $\Delta_{k}$ a set $N_{\sigma, k}$ in $\mathfrak{N}$ such that $\sigma \in \Delta_{k}$ and $x \notin N_{\sigma, k}$ imply

$$
\bigwedge_{\tau \in \Gamma_{k}} f_{\tau}(x) \leqq f_{\sigma}(x) \leqq \bigvee_{\tau \in \Gamma_{k}} f_{\tau}(x) .
$$

Let $\Gamma=\bigcup_{k} \Gamma_{k}$ and $N_{\sigma}=\bigcup_{k} N_{\sigma, k}$. Then $\Gamma$ is countable and cofinal, and $N_{\sigma} \in \mathfrak{R}$. We now define $f_{\sigma}^{*}(x)$ as follows. If $\sigma \in \Gamma$, let $f_{\sigma}^{*}(x)=f_{\sigma}(x)$ for every $x$. If $\sigma \notin \Gamma$ and $x \notin N_{\sigma}$, let $f_{\sigma}^{*}(x)=f_{\sigma}(x)$, and if $\sigma \notin \Gamma$ and $x \in N_{\sigma}$, define $f_{\sigma}^{*}(x)$ $=\lim \inf _{\tau \in \Gamma} f_{\tau}(x)$. Each function $f_{\sigma}^{*}$ is obviously $\mathfrak{B}$-measurable again, and $f_{\sigma}=f_{\sigma}^{*} \bmod \mathfrak{\Re}$.

Consider now any point $x$ in $E$ and any index $\sigma$ in $\Delta_{k}$. If $\sigma \notin \Gamma$ and $x \notin N_{\sigma}$ we have (11.3) and $f_{\sigma}^{*}(x)=f_{\sigma}(x)$, and therefore, since $f_{\tau}=f_{\tau}^{*}$ for every $\tau$ in $\Gamma$,

$$
\bigwedge_{\tau \in \mathrm{r} \Delta_{k}} f_{\tau}^{*}(x) \leqq f_{\sigma}^{*}(x) \leqq \bigvee_{\tau \in \mathrm{r} \Delta_{k}} f_{\tau}^{*}(x) \text {. }
$$

By definition of $f_{\sigma}^{*}$ the same inequalities hold if $\sigma \notin \Gamma$ and $x \in N_{\sigma}$, and finally (11.4) is trivial in the case $\sigma \in \Gamma$. Thus (11.4) is true for every $x$, every $k$, and every $\sigma$ in $\Delta_{k}$, and this implies

$$
\liminf _{\tau \in \Gamma} f_{\tau}^{*} \leqq \liminf _{\sigma \in \Theta} f_{\sigma}^{*}, \quad \limsup _{\sigma \in \Theta}^{*} f_{\sigma}^{*} \leqq \limsup _{\tau \in \Gamma} f_{\tau}^{*} .
$$

Hence $\left(f_{0}^{*}\right)$ is separable.

Let $\left(\mathfrak{B}_{\sigma} ; \sigma \in \Theta\right)$ be a sequence of Boolean sigma-subalgebras of $\mathfrak{B}$ which need not be increasing. A stochastic process $\left(f_{\sigma} ; \sigma \in \Theta\right)$ is called a process relative to $\left(\mathfrak{B}_{\sigma}\right)$ if $f_{\sigma}$ is $\mathfrak{B}_{\sigma}$-measurable for each $\sigma$. Let $\mathfrak{B}_{\sigma}^{*}$ be the Boolean sigmaalgebra of all sets $A+N$ with $A \in \mathfrak{B}_{\sigma}$ and $N \in \Re$, where + denotes the symmetric difference of sets. If $\left(f_{\sigma}\right)$ is a stochastic process relative to $\left(\mathscr{B}_{\sigma}\right)$, the sequence $\left(f_{\sigma}\right)$ constructed in 11.6 is a process relative to $\left(\mathfrak{B}_{\sigma}^{*}\right)$ but it cannot 
always be chosen in such a way that it is a process relative to $\left(\mathfrak{B}_{\sigma}\right)$ again. Therefore it seems reasonable to look for conditions on $\left(\mathscr{B}_{\sigma}\right)$ which are necessary and sufficient in order that every process relative to $\left(\mathfrak{B}_{\sigma}\right)$ is itself separable.

A sequence $\left(K_{\sigma}\right)$ in $\mathfrak{B}$ is called a fine quasicovering of a subset $M$ of $E$ if the following statement is true:

$C^{*}\left(M,\left(K_{\sigma}\right)\right)$. For every index $\sigma$ we have $K_{\sigma} \in \mathfrak{B}_{\sigma}$, and $M \subseteq U\left\{K_{\sigma} ; \sigma \in \Delta\right\}$ for every terminal subset $\Delta$ of $\Theta$.

The sequence $\left(\mathfrak{B}_{\sigma} ; \sigma \in \Theta\right)$ is said to have the property $L$ if:

$L$. Given any fine quasicovering $\left(K_{\sigma}\right)$ of a set $M$ there exists a countable set $\Gamma$ in $\Theta$ with $M \subseteq \cup\left\{K_{\sigma} ; \sigma \in \Gamma\right\} \bmod \mathfrak{N}^{*}$.

The following theorem generalizes the Theorem 4.2 in [8], and its proof is slightly simpler.

11.7. The sequence $\left(\mathfrak{B}_{\sigma}\right)$ satisfies $L$ if and only if every stochastic process relative to $\left(\mathfrak{B}_{\sigma}\right)$ is separable.

Proof. Suppose that every process relative to $\left(\mathfrak{B}_{\sigma}\right)$ is separable, and let $\left(K_{\sigma}\right)$ be a fine quasicovering of a set $M$. Denoting the characteristic function of a set $Q$ by $\chi_{Q}$ we obtain a process $\left(\chi_{K_{\sigma}}\right)$ relative to $\left(\mathfrak{B}_{\sigma}\right)$ with $\chi_{M} \leqq \lim \sup _{\sigma}{ }^{*} \chi_{K_{\sigma}}$. Since $\left(\chi_{K_{\sigma}}\right)$ is separable there is a countable set $\Gamma$ in $\Theta$ with

$$
\chi_{M}=\leqq \limsup _{\sigma \in \Gamma}^{*} \chi_{K_{\sigma}} \bmod \Re^{*},
$$

i.e. $M \subseteq \cup\left\{K_{\sigma} ; \sigma \in \Gamma\right\} \bmod \mathfrak{R}^{*}$.

Suppose now that $L$ holds, and let $\left(f_{\sigma}\right)$ be a process relative to $\left(\mathfrak{B}_{\sigma}\right)$. Furthermore let $\alpha$ be a rational number, $\left\{\Delta_{1}, \Delta_{2}, \cdots\right\}$ a countable basis of the filter of all terminal sets in $\Theta, M_{\alpha}=\left\{x: \lim \sup _{\sigma}{ }^{*} f_{\sigma}(x)>\alpha\right\}$, and $K_{\sigma}$ $=\left\{x: f_{\sigma}(x)>\alpha\right\}$. Then $\left(K_{\sigma}\right)$ is a fine quasicovering of $M_{\alpha}$. Therefore there exists to each $k$ a countable subset $\Gamma_{\alpha, k}$ of $\Delta_{k}$ with $M_{\alpha} \subseteq U\left\{K_{\sigma} ; \sigma \in \Gamma_{k}\right\}$, hence $\mathrm{V}^{*}\left\{f_{\sigma} ; \sigma \in \Gamma_{k}\right\}>\alpha$ in $M_{\alpha} \bmod \mathfrak{R}^{*}$. With $\Gamma=U\left\{\Gamma_{\alpha, k} ; \alpha\right.$ rational, $\left.k=1,2, \cdots\right\}$ we obtain $\bigvee^{*}\left\{f_{\sigma} ; \sigma \in \Delta_{k} \Gamma\right\}>\alpha$ in $M_{\alpha} \bmod \mathfrak{N}^{*}$ for every $k$ and every rational $\alpha$, thus $\lim \sup _{\sigma \in \Gamma}^{*} f_{\sigma} \geqq \alpha$ in $M_{\alpha} \bmod \mathfrak{N}^{*}$ for every rational $\alpha$, and therefore $\lim \sup _{\sigma \in \Gamma}^{*} f_{\sigma} \geqq \lim \sup _{\sigma \in \Theta}^{*} f_{\sigma} \bmod \mathfrak{R}^{*}$. This completes the proof.

We now assume that the sequence $\left(\mathfrak{B}_{\sigma}\right)$ increases. A stochastic process $\left(f_{\sigma} ; \sigma \in \Theta\right)$ is called a semimartingale in the integrand representation relative to $\left(\mathfrak{B}_{\sigma} ; \sigma \in \Theta\right)$ if $f_{\sigma}$ is $\mathfrak{B}_{\sigma}$-measurable and integrable, and $\tau \ll \sigma$ and $A \in \mathfrak{B}_{\tau}$ imply $\int_{A} f_{\tau} d \mu \leqq \int_{A} f_{\sigma} d \mu$. A semimartingale relative to $\left(\mathscr{B}_{\sigma}\right)$ in the integral representation is a sequence $\left(\phi_{\sigma} ; \sigma \in \Theta\right)$ where $\phi_{\sigma}$ denotes a countably additive set function on $\mathfrak{B}_{\sigma}$ such that $\tau \ll \sigma$ and $A \in \mathfrak{B}_{\tau}$ imply $\phi_{\tau}(A) \leqq \phi_{\sigma}(A)$. If $\left(f_{\sigma}\right)$ is a semimartingale relative to $\left(\mathfrak{B}_{\sigma}\right)$ and $\left(f_{\sigma}^{*}\right)$ constructed as in $11.6,\left(f_{\sigma}\right)$ and $\left(f_{\sigma}^{*}\right)$ are semimartingales relative to $\left(\mathfrak{B}_{\sigma}^{*}\right)$ with the same integral representation.

It is now obvious how the definitions and theorems of the Chapters $2-10$ look in the present case. As an example we state the following theorem resulting from 8.1, 11.4, and 11.6. 
11.8. Suppose the sequence $\left(\mathfrak{B}_{\sigma} ; \sigma \in \Theta\right)$ satisfies Vitali's condition $V_{0}$. Then every martingale relative to $\left(\mathfrak{B}_{\sigma} ; \sigma \in \Theta\right)$ of bounded variation from above or from below converges mod $\mathfrak{R}$. If it is separable, it converges also in almost all points of $E$, and its pointwise limit is measurable relative to the completion of $\mu$. To every martingale $\left(f_{\sigma}\right)$ relative to $\left(\mathfrak{B}_{\sigma}\right)$ there exists a separable martingale $\left(f_{\sigma}^{*}\right)$ relative to $\left(\mathfrak{B}_{\sigma}^{*}\right)$ such that $f_{\sigma}=f_{\sigma}^{*} \bmod \mathfrak{R}$ for every $\sigma$.

In the statement about convergence mod $\mathfrak{N}$ the assumption that $\Theta$ has a countable cofinal subset is superfluous, of course.

\section{BIBLIOGRAPHY}

1. E. S. Andersen and B. Jessen, Some limit theorems on integrals in an abstract set, Danske Vid. Selsk. Mat.-Fys. Medd. vol. 22, no. 14 (1946) 29 pp.; Some limit theorems on set-functions, ibid. vol. 25 , no. 5 (1948) 8 pp.

2. S. Bochner, Partial ordering in the theory of martingales, Ann. of Math. (2) vol. 6 (1955) pp. $162-169$.

3. J. Dieudonné, Sur un théorème de Jessen, Fund. Math. vol. 37 (1950) pp. 242-248.

4. J. L. Doob, Stochastic processes, New York, 1953.

5. G. Fichera, Intorno al passaggio al limite sotto il segno d'integrale, Portugaliae Mathematica vol. 4 (1943) pp. 1-20.

6. Haupt-Aumann-Pauc, Differential- und Integralrechnung III, 2d ed., Berlin, 1955.

7. L. L. Helms, Thesis, Purdue University, Lafayette, Indiana, 1956.

8. K. Krickeberg, Extreme Derivierte von Zellenfunktionen in Booleschen $\sigma$-Algebren und ihre Integration, Sitzungsberichte der Bayerischen Akademie der Wissenschaften, MathematischNaturwissenschaftliche Klasse (1955) pp. 217-279.

9. J. M. H. Olmstedt, Lebesgue theory on a Boolean algebra, Trans. Amer. Math. Soc. vol. 51 (1942) pp. 164-193.

10. J. L. Snell, Applications of martingale system theorems, Trans. Amer. Math. Soc. vol. 73 (1952) pp. 293-312.

11. C. de la Vallée Poussin, Sur l'intégrale de Lebesgue, Trans. Amer. Math. Soc. vol. 16 (1915) pp. 435-501.

UNIVERSITY OF ILLINOIS,

URBANA, ILL. 